\title{
Il benessere, un percorso multidisciplinare
}

\author{
A cura di Riccardo Bonato e Marianna Nobile
}

Prefazione di Patrizia Borsellino

Ledizioni 


\author{
(C) 2014 Ledizioni LediPublishing \\ Via Alamanni, 11 - 20141 Milano - Italy \\ www.ledizioni.it \\ info@ledizioni.it \\ Il benessere, un percorso multidisciplinare \\ A cura di Riccardo Bonato e Marianna Nobile \\ Prima edizione: ottobre 2014 \\ ISBN cartaceo 978-88-6705-259-2 \\ ISBN ebook 978-88-6705-260-8 \\ Copertina e progetto grafico: ufficio grafico Ledizioni
}

Informazioni sul catalogo e sulle ristampe dell'editore: www.ledizioni.it

Le riproduzioni a uso differente da quello personale potranno avvenire, per un numero di pagine non superiore al $15 \%$ del presente volume, solo a seguito di specifica autorizzazione rilasciata da Ledizioni.

Volume finanziato con il bando "1000 lire" dell'Università degli Studi MilanoBicocca 


\section{Indice}

PREMESSA

di Patrizia Borsellino

INTRODUZIONE

VII

di Marianna Nobile e Riccardo Bonato

DIRITTO TRIBUTARIO E BENESSERE SOCIALE

di Leda Rita Corrado

STRUTTURE MATEMATICHE PER IL BENESSERE:

Il TEOREMA DI ARROW

di Simone Camosso

DIRITTO ALLA SALUTE, DISEGUAGLIANZE E MATERIALITÀ DEL SOCIALE: UNA RICOGNIZIONE PER LEGGERE LE MOLTEPLICI CORRELAZIONI TRA DIRITTO E SOCIETÃ NELL'AREA DEL BENESSERE:

di Carlo Botrugno

MUTILAZIONI GENITALI FEMMINILI: IL CONFLITTO

TRA DIRITTI FONDAMENTALI E TRADIZIONI CULTURALI

di Marianna Nobile

I CONTRATTI FLESSIBILI E IL BENESSERE FAMIGLIARE.

CASO DI STUDIO: LE SCUOLE DELL'INFANZIA

DEL COMUNE DI MILANO

59

di Riccardo Bonato e Chiara Caulo

BIOGRAFIA DEGLI AUTORI 


\section{PREMESSA}

\section{Patrizia Borsellino*}

I contributi che il lettore trova raccolti in Il benessere, un percorso multidisciplinare, traggono origine da un'interessante iniziativa che ha visto coinvolti laureandi e, soprattutto, dottorandi, che stanno compiendo il loro percorso formativo, in vista del conseguimento del Dottorato di ricerca, nell'Università di Milano-Bicocca e nell'Università di Bologna. L'iniziativa in questione, promossa dai curatori del volume, Marianna Nobile e Riccardo Bonato, entrambi dottorandi in Scienze giuridiche, nell'ambito del curriculum in Filosofia e Sociologia del diritto dell'Università di Milano-Bicocca, è consistita nella realizzazione, nella prima metà del 2014, di una serie di seminari pensati con l'intento di favorire l'incontro e il confronto di giovani studiosi su un tema, quello del "benessere", di comune interesse, ma, abitualmente, affrontato da ognuno dall'angolo visuale e con gli strumenti propri del rispettivo ambito disciplinare. Quella di "benessere", nelle sue diverse articolazioni e specificazioni di benessere fisico, psicologico, sociale, economico, è una nozione che riveste un ruolo centrale nelle riflessioni bioetiche sui modi appropriati dell'erogazione delle cure e sulla preferibilità e fattibilità dei diversi modelli di assistenza sanitaria, così come nelle indagini giuslavoristiche e sociologico-giuridiche sulle ripercussioni, a livello individuale e sociale, dell'organizzazione del lavoro, oppure negli studi sui sistemi tributari in grado di conciliare, al meglio, equità ed efficacia. Ma si tratta di nozione che, soprattutto nell'accezione di

\footnotetext{
* Professore ordinario di filosofia del diritto e di bioetica, Direttore del Dipartimen-
} to dei Sistemi Giuridici dell'Università degli Studi di Milano-Bicocca 
benessere sociale, è oggetto di sempre maggiore attenzione anche da parte di matematici impegnati nell'elaborazione di modelli di scelta razionale. I saggi realizzati a partire da alcune delle relazioni presentate nei seminari forniscono uno sguardo d'insieme di alcune tra le più significative prospettive dalle quali il tema del benessere si presta ad essere affrontato, ed appaiono meritevoli di apprezzamento. A renderli tali non sono però solo gli interessanti elementi di analisi che contengono. E', anche e soprattutto, la circostanza di essere stati realizzati da giovani studiosi disponibili a guardare oltre i confini della propria disciplina, non certo per invadere il campo altrui, ma per poter sempre meglio coltivare il proprio. Una strada, quella del confronto multidisciplinare, nella quale v'è da auspicare che non solo sappiano procedere coloro che, in modo assai promettente, già l'hanno imboccata, ma si incamminino anche coloro che, giovani e soprattutto meno giovani, non sembrano talora capaci di spingere lo sguardo oltre il loro recinto. 


\section{INTRODUZIONE}

Marianna Nobile e Riccardo Bonato

La riflessione intorno al concetto di benessere trova origine nella filosofia antica, quando Aristotele considerò l'eudaimonia, intesa come perfezione individuale e come attuazione delle proprie capacità, il fine ultimo dell'esistenza umana. Nel dibattito contemporaneo, seppur sia possibile riscontrare un accordo per lo più unanime in merito alle caratteristiche che, in via generale, deve avere una "vita buona", è difficile individuare un'univoca definizione di benessere. Nel rapporto della Commissione Salute dell'Osservatorio europeo sui sistemi e le politiche per la salute, a cui partecipa il distaccamento europeo dell'Organizzazione Mondiale della Sanità (OMS), è stata proposta una definizione di benessere come «lo stato emotivo, mentale, fisico, sociale e spirituale di ben-essere che consente alle persone di raggiungere e mantenere il loro potenziale personale nella società $>^{1}$.

Tuttavia, per poter cogliere appieno le molteplici sfaccettature del concetto di benessere, è indispensabile prendere in considerazione le diverse dimensioni che questo assume. Si possono, infatti, distinguere sia una dimensione "soggettiva", basata sulla valutazione individuale delle proprie condizioni di vita; sia una dimensione "oggettiva", connessa agli aspetti materiali, per lo più socio-economici,

1 «Wellness is a state of emotional, mental, physical, social and spiritual wellbeing that enables people to reach and maintain their personal potential in their communities», Working Together for Wellness. A Wellness Strategy for New Brunswick, Second Report of the Select Committee on Health Care, Third Session Fifty-fourth Legislative Assembly of the Province of New Brunswick, April 2001. 
in virtù dei quali è possibile raggiungere o mantenere uno stato di benessere. Nella prospettiva soggettiva, che considera il benessere in chiave individuale, emozionale, nonché psicologica, emergono due diversi orientamenti. Da una parte, la tradizione edonica riferisce il benessere principalmente alla dimensione affettiva e alla soddisfazione personale, prestando particolare attenzione a ciò che rende le esperienze e la vita piacevoli o spiacevoli ${ }^{2}$. Dall'altra parte, l'approccio eudaimonico o cognitivo supera il concetto di felicità, intendendo il benessere come generato dalla realizzazione della propria vera natura e come il risultato del raggiungimento di obiettivi positivi ${ }^{3}$.Alcuni studiosi hanno, dunque, proposto di definire il benessere come «la valutazione globale della qualità della vita degli individui in base ai criteri da questi scelti» ${ }^{4}$. Il concetto di "qualità della vita" viene definito, secondo l'OMS come «la percezione individuale della propria posizione nella vita in riferimento al contesto culturale e al sistema di valori di appartenenza e in relazione ai propri obiettivi, aspettative, standard e preoccupazioni. È un concetto di ampia portata influenzato in modo complesso dalla salute fisica, dallo stato psicologico, dalle credenze personali, dalle relazioni sociali e dalle condizioni ambientali $>^{5}$. La dimensione soggettiva del benessere, quindi, viene ricondotta principalmente alle percezioni individuali. Essa rappresenta il processo attraverso il quale ciascun individuo valuta la propria vita, facendo riferimento a determinati standard personali. Tale consapevolezza consente di esprimere un livello di soddisfazione in funzione del raggiungimento dei propri obiettivi, della realizzazione delle proprie aspirazioni, del confronto con i propri ideali e con le proprie esperienze passate.

L'approccio basato sulla dimensione oggettiva del benessere, invece, fa uso di indicatori economici e sociali che rispecchiano le cir-

\footnotetext{
2 Cfr. Kahneman D., Diener E., Schwarz N. (a cura di), Well-being: Foundations of hedonic psychology, Russell Sage Foundation Press, New York, 1999.

3 Waterman A.S., Two conceptions of happiness: Contrasts of personal expressiveness (eudaimonia) and hedonic enjoyment, in Journal of Personality and Social Psychology, 64(4), 1993, pp.678-691.

4 Shin D., Johnson D, Avowed happiness as an overall assessment of the quality of life, in Social Indicators Research, 5(1), 1978, p. 478.

5 «Quality of life is an individual's perception of their position in life in the context of the culture and value systems in which they live and in relation to their goals, expectations, standards and concerns. It is a broad ranging concept affected in a complex way by the person's physical health, psychological state, personal beliefs, social relationships and their relationship to salient features of their environment». World Health Organization, WHOQOL Measuring Quality of Life, Geneva, 1997.
} 
costanze oggettive in una determinata area geografica o culturale ${ }^{6}$. La peculiarità di tali indicatori si riscontra nel fatto che essi fanno riferimento ad analisi statistiche e quantitative, piuttosto che alle percezioni individuali. Questi vengono prevalentemente utilizzati in ambito politico-governativo al fine di analizzare lo stato di un fenomeno sociale e di individuare gli obiettivi da perseguire. In questo modo gli indicatori assumono carattere normativo al fine della valutazione di politiche volte al miglioramento delle condizioni socio-economiche di una determinata popolazione. A muovere dalla diffusa convinzione che il benessere dipenda da determinanti socioeconomici e che possa essere misurato esclusivamente in termini quantitativi, a partire dagli anni '60 del secolo scorso, negli USA si è assistito allo sviluppo del cosiddetto "movimento degli indicatori sociali", sorto in relazione all'esigenza della pubblica amministrazione di disporre informazioni sullo stato della società al fine di creare preformance budgets relativamente ai bisogni sociali essenziali, quali, ad esempio, salute, istruzione, abitazione, lavoro, etc ${ }^{7}$.

Tuttavia, nel dibattito contemporaneo diversi studiosi ritengono che la misurazione del benessere debba essere necessariamente affiancata dall'approccio psicologico volto a rivalutare l'esperienza individuale, in base al quale, come precedentemente osservato, ciascun individuo formula un giudizio di valore sulla propria esperienza diretta ${ }^{8}$. Già nel 1934, l'economista Simon Kuznets ammise che gli indicatori economici erano solo una componente del benessere dei cittadini e che «il benessere di una nazione può [...] difficilmente essere desunto dalla misurazione delle entrate nazionali $>^{9}$. Si pensi, ad esempio, che, nonostante negli USA il PIL sia triplicato nell'arco di 50 anni, il benessere dei singoli cittadini è rimasto immutato, facendo emergere, al contrario, un drammatico aumento della percentuale di individui affetti da ansia e depressione ${ }^{10}$. Si rileva, dunque, che la sovrabbondanza di beni e servizi disponibili nei Paesi più sviluppati non riflette il benessere soggettivo dei cittadini e che quest'ultimo non dipende meramente da circostanze oggettive, ma

\footnotetext{
$6 \quad$ Cfr. Land K.C., Social indicators and the quality of life: Where do we stand in the mid1990s?, in SINET (Science Information Network), 45, 1996, pp. 5-8.

7 National Commission on Technology, Report, 1966, Washington (D.C.)

8 Di Franco G., Qualità della vita: dai modelli alle ricerche empiriche, in Vergati S. (a cura di), Dimensioni sociali e territoriali della qualità della vita, La Goliardica, Roma, 1989.

9 Kuznets S., National income 1929-1392, Senate document No. 124, 73rd US Congress, 1934, p.7.

10 Forgeard M.J.C. et al., Doing the right thing: Measuring wellbeing for public policy, in International Journal of Wellbeing, 1(1), 79-10.
} 
anche e soprattutto dalle esperienze e dai bisogni propri del singolo individuo.

Alla luce di queste considerazioni si può ritenere che il benessere sia una condizione in cui la componente soggettiva e quella oggettiva si condizionano vicendevolmente e che tale mutua relazione costituisca il risultato di un percorso complesso di cui si dovrà tener conto se si intende analizzare il benessere degli individui per come è effettivamente percepito e valutato dagli stessi. Lo scopo della seguente raccolta di saggi sarà, dunque, di analizzare particolari aspetti del concetto di benessere, tenendo conto delle dimensioni sociali che questo può assumere. Nel primo contributo si osserverà come, in riferimento al contesto nazionale, il sistema tributario non debba essere considerato come fine a se stesso, ma piuttosto come il mezzo attraverso il quale la collettività può prendere parte al mantenimento del benessere sociale. Tale scopo è, infatti, chiaramente stabilito nei principi della Costituzione Italiana, tra i quali è opportuno ricordare il dovere di solidarietà economica (art. 2, Cost.), il principio di capacità contributiva (art. 53, comma 1, Cost.), il principio di progressività (art. 53, comma 2, Cost.), la riserva di legge (art. 23, Cost.) e il principio della parità di bilancio (art. 81, Cost.).

Successivamente, verrà preso in considerazione un ulteriore strumento utilizzabile per il raggiungimento del benessere sociale, ovvero la modellizzazione matematica del dilemma di scelta, applicata al concetto di benessere collettivo, dimostrando come quest'ultimo dipenda da funzioni di benessere individuale connesse alle scelte delle singole persone. Nello specifico sarà approfondito il teorema di Arrow, il quale, analizzando il modello democratico come mezzo di aggregazione delle scelte individuali per una decisione collettiva, dimostra che non può esistere una funzione di scelta sociale indipendente da una funzione di scelta individuale.

Il terzo contributo affronterà il concetto di benessere in ambito sanitario alla luce della definizione di salute proposta dall'OMS, secondo la quale la salute corrisponde a «uno stato di completo benessere fisico, psichico e sociale e non semplice assenza di malattia $\gg^{11}$. Ci si interrogherà, dunque, in merito alla validità e all'efficacia del

11 «Health is a state of complete physical, mental and social well-being, and not merely the absence of disease or infirmity». Dal Preambolo della Costituzione dell'Organizzazione Mondiale della Sanità, adottata dalla Conferenza Mondiale sulla Salute, tenutasi a New York il 22 luglio 1946 dai rappresentanti di 61 Stati (Official Records of the World Health Organization, no. 2, p. 100) e entrata in vigore il 7 aprile 1948. La definizione di salute non è stata modificata dal 1948. 
diritto alla salute, sancito dall'art. 32 della Costituzione Italiana ${ }^{12}$, nonché relativamente all'opportunità di definire un "nucleo irriducibile" di protezione, necessario per orientare le risorse delle Stato in modo efficiente.

Verrà, quindi, introdotto il concetto di vulnerabilità delle minoranze quale limite alla protezione giuridica e al godimento di beni primari, approfondito, successivamente, attraverso l'analisi di due esempi di minoranze: i migranti e i lavoratori atipici. L'attuale configurazione multiculturale della società occidentale impone, infatti, che ci si interroghi sulle diverse modulazioni della relazione di cura e sulle richieste avanzate da individui appartenenti a comunità, le cui tradizioni spesso risultano contrastare con i valori liberali propri delle società ospitanti. In questo contesto si inserisce il tema delle mutilazioni genitali femminili, che diventano un problema quando la richiesta di effettuare tali pratiche viene avanzata da alcuni gruppi sociali radicati nel contesto occidentale, in quanto considerata come un'istanza volta a fare il "bene" di chi ne è destinatario. Ci si domanderà, dunque, fino a che punto tali pretese siano accoglibili e giustificabili, qualora siano in aperto contrasto con la tutela della dignità e dell'integrità personale, elementi considerati indispensabili ai fini del raggiungimento di un completo stato di benessere individuale.

Tuttavia, come verrà osservato nell'ultimo contributo, le minoranze che difficilmente trovano tutela dei loro diritti possono essere generate non solo da fattori etnici e culturali. Ne sono un esempio i lavoratori atipici, altresì detti "flessibili", in quanto assunti con contratti diversi dal contratto a tempo indeterminato. Il fattore che crea la minoranza è, in questo caso, il contratto di lavoro, il quale, attraverso componenti economiche e psicologiche, impatta su dimensioni ulteriori al mondo lavorativo, quali la dimensioni individuale e famigliare. Infatti, la vulnerabilità del lavoratore "flessibile" si estende oltre alla continua mutevolezza del suo orizzonte lavorativo (mansioni e luogo di lavoro) e al rischio di instabilità reddituale, che si riversa sulle scelte di vita dei soggetti e delle loro famiglie. Una recente ricerca quantitativa ha mostrato come la tipologia di contratto di lavoro della madre possa esercitare almeno un parziale

\footnotetext{
12 «La Repubblica tutela la salute come fondamentale diritto dell'individuo e interesse della collettività, e garantisce cure gratuite agli indigenti.

Nessuno può essere obbligato a un determinato trattamento sanitario se non per disposizione di legge. La legge non può in nessun caso violare i limiti imposti dal rispetto della persona umana». Costituzione della Repubblica Italiana, art. 32.
} 
effetto sullo sviluppo linguistico del figlio, nella misura in cui il tipo di contratto di lavoro influenza la possibilità di usufruire delle tutele alla maternità (nel caso specifico i permessi di lavoro giornalieri per l'allattamento). Questo è un esempio di come il benessere delle generazioni future possa dipendere dal livello di protezione giuridica che la nostra società riesce ad accordare a minoranze con particolari vulnerabilità. 


\title{
DIRITTO TRIBUTARIO E BENESSERE SOCIALE
}

\author{
LEDA Rita CORRADO*
}

Nel sentire comune il diritto tributario è percepito in maniera estremamente negativa. Questa affermazione trova riscontro anche nell'osservazione empirica: significativi sono gli episodi che possono essere tratti dalla cronaca quotidiana. È vero che la crisi economica ${ }^{1}$ ha accentuato questa percezione: ciononostante il sentimento di avversione che suscita la fiscalità nell'uomo medio è una costante, come confermano storia e letteratura. Quale efficace esempio tratto dalla prima è sufficiente richiamare alla mente le parole di Tacito: ne La Germania ${ }^{2}$, egli ricorda che, come segno dell'antica alleanza con i Romani, alla popolazione germanica dei Batavi spetta «un glorioso privilegio» perché «non subiscono l'umiliazione dei tributi né le vessazioni degli esattori: esenti da gravami e contribuzioni, sono serbati per il solo utilizzo in battaglia, come armi di offesa e di difesa». Dalla seconda, come non menzionare le parole di Jacques Prévert ${ }^{3}$ : «Le ministère des Finances devrait s'appeler ministère de la Misère puisque le ministère de la Guerre ne s'appelle pas ministère de la Paix». Lo stesso Luigi Einaudi ${ }^{4}$ scriveva

\footnotetext{
* Dottoranda di ricerca in Diritto Tributario

Dipartimento dei Sistemi Giuridici dell'Università degli Studi di Milano-Bicocca

1 Nel rapporto How's Life? 2013 dell'OCSE si legge che il grado di soddisfazione degli Italiani in merito alle proprie condizioni di vita è diminuito del $12 \%$ tra il 2007 e il 2012, mentre il Bel Paese è scivolato al $29^{\circ}$ posto in una graduatoria che vede coinvolti (solo) 35 stati (OCSE, How's life? 2013 - Measuring well-being, 2013, http:// www.oecd.org/statistics/howslife.htm).

2 Tacito, De origine et situ Germanorum (98 d.C.), trad. it., La Germania, Mondadori, Milano, 1991, § 29.

3 J. Prévert, Histoires, 1963.

4 L. Einaudi, Miti e paradossi della giustizia tributaria, Einaudi, Torino, 1938.
} 
«Gli economisti ebbero nel secolo scorso il torto di aggravare la propensione ad interpretare la parola "imposta" nel senso di peso o di dolore con la malaugurata collocazione che essi fecero, per ragioni di euritmia architettonica, della discussione delle imposte nella quarta parte dei loro trattati. Produzione, distribuzione, circolazione e consumazione della ricchezza: ecco la classica quadripartizione del dramma economico [...]. I primi tre atti del dramma erano gli atti creativi. [...] Poi veniva il diluvio. Il consumo distruggeva e, nell'atto di consumo, aveva luogo una lotta intestina tra gli uomini, i quali pretendevano di consumare tutto quello che avevano prodotto, e lo Stato il quale voleva portare via loro una parte per provvedere ai consumi pubblici. Due idee-forza si sprigionano da questa architettura accademica: che l'imposta sia distruzione e che essa sia distruzione di quel che altri, ha creato».

Anche se le espressioni «benessere» e «diritto tributario» suonano ai più dissonanti, questo stridore è solo apparente. Per tentare di dimostrare ciò, si può partire dal loro significato.

Il «benessere» è definito dal vocabolario Treccani.it come «stato felice di salute, di forze fisiche e morali», "condizione prospera di fortuna, agiatezza» ovvero «sensazione soggettiva di vita materiale piacevole». Il concetto di «benessere» è uno dei capisaldi delle scienze economiche, declinato sia nella sua dimensione individuale (cfr. curve di indifferenza in microeconomia) sia in quella collettiva (cfr. l'economia del benessere nel pensiero di Pigou e Pareto).

Garantire il benessere sociale è lo scopo del «diritto tributario»: questo settore dell'ordinamento giuridico riunisce le regole in base alle quali la ricchezza dei consociati viene trasferita alle organizzazioni pubbliche cui è demandato il compito di fornire servizi alla collettività (sanità, istruzione, sicurezza, difesa ecc.). Quando si vuole spiegare questa definizione al di fuori della ristretta cerchia dei cultori della materia, solitamente si ricorre a similitudini.

«La spesa del governo è, in riguardo agli individui di una grande nazione, come la spesa d'amministrazione riguardo ai comproprietari di un grande patrimonio, i quali sono tutti obbligati di contribuirvi in proporzione ai loro rispettivi interessi nel medesimo».

Così scriveva Adam Smith ${ }^{5}$. Un frequente accostamento è quello tra sistema tributario e condominio. Questa scelta è forse quella più vicina alla realtà delle cose (si pensi ai numerosi casi di amministratori infedeli che allietano le giornate dei condomini vittime delle

\footnotetext{
5 A. Smith, An Inquiry into the Nature and Causes of the Wealth of Nations, 1776, trad. it., Ricerche sopra la natura e le cause della ricchezza delle Nazioni, Mondadori, Milano, 1977.
} 
loro ruberie), ma porta con sé un retrogusto amaro. Più rasserenante sembra il paragone con gli amici in gita che, per spuntare condizioni economicamente più vantaggiose, acquistano i biglietti del treno come gruppo anziché singolarmente. Allo stesso modo i consociati destinano parte della propria ricchezza al finanziamento di servizi comuni.

Tra contribuenti e organizzazioni pubbliche esiste un rapporto sinallagmatico: sui primi incombe un dovere inderogabile di solidarietà economica, mentre sulle seconde grava il compito di garantire il benessere della collettività (cfr. art. 2 Cost.). Luigi Einaudi ${ }^{6}$ affermava che

«Mercé l'imposta, lo Stato crea l'ambiente giuridico e politico nel quale gli uomini possono lavorare, organizzare, inventare produrre. [...] Non perciò si afferma che tutto il prodotto sociale, tutto il reddito nazionale sia di spettanza dello Stato. Si afferma soltanto che esiste una distribuzione del reddito nazionale annuo che è l'ottima fra tutte: una distribuzione grazie alla quale lo Stato riceve l'imposta, il lavoratore il salario, il risparmiatore l'interesse, l'imprenditore il profitto e il proprietario la rendita; ed ognuno riceve quel che è suo, quel che fu creato da lui, quel che è necessario egli abbia affinché la sua partecipazione all'opera comune sia la massima e la più efficace».

\section{Esprimeva la medesima linea di pensiero anche Ezio Vanoni ${ }^{7}$ :}

«Organizzazione economica ed organizzazione politica raggiungono il proprio fine quando creano le condizioni perché l'uomo sia se stesso e possa attuare il proprio destino di perfezione in piena responsabilità e libertà. Le vie per la liberazione dell'individuo dalla miseria e dagli ostacoli materiali che lo inceppano sono di tempo in tempo diversi. Ma il fine di ogni azione nella società resta per noi immutabile: fare in modo che ogni uomo possa liberamente tendere a realizzare la pienezza di vita che risponde alla sua natura, e alla chiamata divina che lo sospinge».

\section{E ancora secondo Vanoni ${ }^{8}$ :}

«Dalla Rivoluzione Francese e dalla successiva rielaborazione di dottrine e di istituti sorge la moderna imposta. Giusto contemperamento delle imposte indirette e dirette, rispetto dei redditi minimi, rapporto diretto alle ricchezze dei singoli, la caratterizzano da un punto di vista economico: i canoni della certezza dei tributi e della giustizia tributaria, il requisito dell'approvazione delle leggi di finanza da par-

\footnotetext{
6 L. Einaudi, Miti e paradossi della giustizia tributaria, Einaudi, Torino, 1938.

7 E. Vanoni, La nostra via. Criteri politici dell'organizzazione economica, Seli, Roma, 1947.

8 E. Vanoni, Natura ed interpretazione delle leggi tributarie, Cedam, Padova, 1932.
} 
te degli organi rappresentativi, mentre la garantiscono dall'arbitrio, la identificano dal punto di vista giuridico: la relazione di necessità colla esplicazione dell'attività pubblica la giustificano in linea etica. Ora come è possibile ritenere ancora che un istituto il quale si presenta con questi caratteri, abbia in sé tali elementi da dover essere inteso sempre in senso contrario all'interesse dello Stato?».

Si dirà che queste sono soltanto speculazioni teoriche, per di più piuttosto datate. La drammatica attualità di tali considerazioni è invece confermata da un significativo episodio avvenuto negli U.S.A. nell'ottobre 2013, quando sul sito della Casa Bianca è stato inserito un avviso dal seguente tenore: «Because Congress did not fulfil its responsibility to pass a budget, much of the federal government is shut down». Il mancato accordo sul bilancio tra il Presidente Obama e i due rami del Parlamento - uno a maggioranza repubblicana, l'altro a maggioranza democratica - ha causato la chiusura (il cosiddetto shutdown) di tutti gli uffici federali ritenuti non essenziali, determinando di fatto la sospensione di molti servizi per i cittadini. Nessun (accordo politico sul) finanziamento, nessun servizio pubblico.

L'obiezione è inoltre tanto facile quanto infondata, non soltanto in ragione dell'indiscussa autorevolezza degli studiosi che hanno formulato le riflessioni sopra citate, ma anche per il ruolo che essi hanno concretamente rivestito nella vita politica italiana. Uno dei personaggi che ha maggiormente inciso sull'evoluzione del sistema tributario italiano - apportando il proprio contributo sia attraverso la propria attività scientifica sia con l'azione di governo - è proprio Ezio Vanoni (Morbegno 3 agosto 1903 - Roma 16 febbraio 1956). Valtellinese, nel 1925 si laurea in giurisprudenza discutendo una tesi su natura e interpretazione delle leggi tributarie sotto la guida di Benvenuto Griziotti, il fondatore della cosiddetta "Scuola di Scienze delle Finanze di Pavia". Dedica gli anni successivi alla docenza universitaria e alla ricerca scientifica, pubblicando svariate monografie, tra le quali in particolare si segnalano «Natura ed interpretazione delle leggi tributarie» (1932), «Osservazioni sul concetto di reddito in finanza» (1932), «La dichiarazione tributaria e la sua irretrattabilità» (1937), «Lezioni di diritto finanziario e scienza delle finanze» (1937), «Problemi dell'imposizione degli scambi» (1939), «L'imposta personale sul reddito e gli utili di società non distribuiti» (1943). Tra il 1943 e il 1944 contribuisce all'elaborazione del programma di politica economica del cattolicesimo sociale enunciati nel cosiddetto «Codice di Camaldoli». Nel 1946 si apre la sua stagione politica. Eletto nell'Assemblea Costituente, partecipa ai lavori della «Com- 
missione dei 75» (così denominata perché composta da 75 deputati in rappresentanza delle forze politiche elette nell'Assemblea) per la stesura del progetto di carta costituzionale da sottoporre all'approvazione dell'Assemblea, contribuendo in particolare alla formulazione delle norme in materia tributaria:

- l'art. 23 Cost., ove si pone il principio di riserva di legge per le prestazioni patrimoniali imposte, dando indiretta applicazione al principio di uguaglianza ex art. 3 Cost.

Art. 23 - Nessuna prestazione personale o patrimoniale può essere imposta se non in base alla legge.

- l'art. 53 Cost., con il quale si cristallizzano il principio di capacità contributiva (comma 1) e il principio di progressività del sistema tributario (comma 2): il primo identifica nelle manifestazioni oggettive di ricchezza (reddito, patrimonio e consumo) e pone un limite al dovere di solidarietà sociale gravante sui singoli consociati nel concorso al finanziamento delle spese pubbliche ex art. 2 Cost., mentre il secondo, in ossequio al principio di uguaglianza sostanziale ex art. 3, comma 2, Cost., fa sì che i tributi nel loro insieme siano disegnati in maniera tale da gravare in misura superiore sui contribuenti che manifestano una maggiore capacità contributiva (in concreto, un tributo può dirsi progressivo quando l'aliquota aumenta con il crescere della base imponibile), così promuovendo la rimozione degli ostacoli che impediscono il pieno sviluppo della persona nell'ambito della comunità sociale.

Art. 53 - 1. Tutti sono tenuti a concorrere alle spese pubbliche in ragione della loro capacità contributiva. 2. Il sistema tributario è informato a criteri di progressività.

- l'art. 81 Cost., che - nel suo testo originario (cfr. modifiche introdotte dall'art. 1, 1. cost. 20 aprile 2012, n. 1) - vieta di stabilire nuovi tributi e nuove spese con la legge di bilancio (comma 3) e pone a garanzia del pareggio di bilancio l'obbligo di indicare i mezzi di copertura idonei a far fronte a nuove e maggiori spese determinate da altra legge (comma 4)

Art. 81 Cost. -1. Le Camere approvano ogni anno i bilanci e il rendiconto consuntivo presentati dal Governo. 2. L'esercizio provvisorio del bilancio non può essere concesso se non per legge e per periodi non superiori complessivamente a quattro mesi. 3. Con la legge di approvazione del bilancio non si possono stabilire nuovi tributi e nuove spese. 4. Ogni altra legge che importi nuove e maggiori spese deve indicare i mezzi per farvi fronte.

Senatore per la Democrazia Cristiana dal 1948, ricopre il ruolo di 
Ministro delle Finanze (1948-1954), Ministro del Tesoro ad interim (1951-1952) e Ministro del Bilancio (1954-1956). Nel 1956 muore dopo aver pronunciato un discorso a Palazzo Madama.

Durante la sua attività politica Ezio Vanoni avvia una riforma strutturale del sistema tributario finalizzata al miglioramento dei rapporti tra consociati e Stato e al risanamento dell'Erario, depauperato non soltanto dalle vicende belliche ma anche - e forse soprattutto - dall'atavica propensione dei contribuenti a sottrarsi al pagamento dei tributi: in un discorso che conserva intatta la sua attualità, Ezio Vanoni ${ }^{9}$ osserva che

\begin{abstract}
«un certo declassamento della moralità fiscale, [...] una legislazione spesso caotica e talvolta ispirata a finalità demagogiche irraggiungibili hanno aggravato notevolmente il fenomeno della evasione fiscale. Fenomeno che oggi [1949 - N.d.A.] si verifica su di una scala preoccupante e che compromette una equa distribuzione dei carichi tributari. In una simile situazione la pressione tributaria diviene vessatoria e veramente insopportabile per gli onesti e per le categorie dei contribuenti che non possono sfuggire all'esatta determinazione dell'imposta per motivi tecnici. [...] La evasione [...] assume i caratteri di uno strumento di concorrenza sleale, così da compromettere i normali rapporti economici e da spingere sulla strada della frode fiscale una schiera sempre più numerosa di contribuenti. [...] Nello Stato moderno, di fronte alla concezione che tutti abbiamo della società e del dovere primo del cittadino di dare la sua solidarietà all'ordinato svolgersi della vita civile [...] l'imposta non può essere intesa che come l'espressione del dovere morale e civico, che grava su ognuno di noi, di concorrere al bene della società. Sottrarsi a questo dovere assume le caratteristiche di una vera e sostanziale forma di anarchia, di una negazione delle esigenze prime della convivenza sociale; è come disertare, sicché allo stesso modo con cui circondiamo di disprezzo il disertore che si rifiuta di difendere il proprio Paese di fronte al nemico, così dovremmo circondare di disprezzo l'evasore tributario, quando il tributo fosse equo».
\end{abstract}

Consapevole della necessità di dover perseguire i propri obiettivi in maniera graduale, mettendo in atto «una sistemazione transitoria [...]senza compromettere eccessivamente il gettito delle imposte e senza mettere in pericolo il bilancio nel periodo di transizione" e agendo "con energia, ma con prudenza, frenando impazienze per quanto legittime» ${ }^{10}$, Ezio Vanoni avvia la sua paziente opera di riforma agendo su più fronti, non soltanto attraverso la correzione della disciplina legislativa (come avvenne, ad esempio, con l'approvazione della tariffa do-

\footnotetext{
E. Vanoni, Relazione al disegno di legge presentato al Senato della Repubblica, seduta del 26 luglio 1949.

10 Ibid.
} 
ganale nel 1950) ma anche risolvendo problemi pratici.

L'intervento più significativo è rappresentato dalla generalizzazione della dichiarazione annuale e unica di tutti i redditi con la 1. 11 gennaio 1951, n. 25, recante «Norme sulla perequazione tributaria e sul rilevamento fiscale straordinario»: con la reintroduzione (cfr. d.lgs. luogotenenziale 24 agosto 1945, n. 585) di questo adempimento periodico ha infatti inizio un progressivo cambiamento nel ruolo di entrambi i soggetti coinvolti nel rapporto tributario. Se dall'Unità d'Italia al Secondo Dopoguerra il contribuente è gravato soltanto dell'obbligo di pagare le somme calcolate dall'Amministrazione finanziaria, a partire dagli Anni Cinquanta egli è chiamato a liquidare il tributo dovuto predisponendo allegando e conservando una dettagliata documentazione dei fatti fiscalmente rilevanti. Per far fronte al controllo di una maggiore mole di adempimenti, è avviato un parallelo processo di riorganizzazione dell'Amministrazione finanziaria, realizzata mediante interventi mirati su sedi (cfr. la razionalizzazione della distribuzione territoriale degli uffici), mezzi (cfr. l'introduzione del meccanografico) e persone (cfr. i corsi di formazione per migliorare la preparazione dei funzionari).

La cosiddetta «Riforma Vanoni» ha assunto una portata quasi pedagogica perché, abituando milioni di contribuenti a interagire regolarmente con l'Amministrazione finanziaria, ha preparato il campo alla tassazione di massa avviata negli Anni Settanta. Nella visione vanoniana ciò che deve mutare non è però soltanto la modalità mediante la quale è assolto l'onere tributario o l'organizzazione della Pubblica Amministrazione, ma anche - e soprattutto - la mentalità dei consociati: Ezio Vanoni ${ }^{11}$ vuole che l'Italia passi da un sistema fiscale in cui l'imposta è... «imposta» e «si paga bestemmiando lo Stato» ad un nuovo assetto che consenta al contribuente di essere conscio della propria «dignità di partecipe della vita statale [nonché del fatto di] esercitare, pagando, una vera e propria funzione sovrana». Per usare le parole pronunciate dallo stesso giurista in una delle riunioni preliminari alla costituzione dell'Associazione Nazionale Tributaristi Italiani (ANTI) tenutasi a Roma nel 1949, il contribuente

«è legato alla vita dello Stato impositore da una sostanziale identità di interessi: la stessa vita dello Stato e la partecipazione dei cittadini alla vita dello Stato, perché il tributo costituisce fattore di giustizia sociale e in questa identità trova la sua giustificazione in una funzione perequativa».

${ }_{11}$ E. Vanoni, Natura ed interpretazione delle leggi tributarie, op. cit. 
Secondo Vanoni ${ }^{12}$ i tributi sono un mezzo indispensabile dell'agire collettivo, attraverso il quale lo Stato può operare

«come organo della stessa sovranità popolare, come espressione genuina dell'autogoverno nazionale. Parlare di odiosità del tributo in sé significa [...] disconoscere l'indissolubile vincolo corrente tra esistenza dello Stato ed imposizione. [...] L'interesse generale non è la somma degli interessi individuali dei membri della collettività, ma è la risultante degli interessi dei singoli modificati e foggiati dalla convivenza nella società nazionale. [...] L'interesse del contribuente, ad ottenere il massimo di utilità individuale col minore sacrificio, non ha alcuna diretta tutela giuridica: esso si risolve nell'accentuazione dell'interesse a partecipare alla vita pubblica, in guisa da influire sul modo di essere dello Stato».

\section{Nella concezione vanoniana ${ }^{13}$, l'utilità sociale}

«è l'utilità di tutti i componenti la società, e soprattutto l'utilità di coloro che non sono in condizioni di contribuire col proprio sacrificio a sostenere la spesa pubblica, perché proprio costoro per la loro debolezza, per la loro incapacità ad appropriarsi la parte di beni che è necessaria per consentire ad essi condizioni di vita degne della loro natura di uomini, sono prima di ogni altro i naturali destinatari dell'azione pubblica».

La rivoluzione copernicana promossa da Ezio Vanoni si traduce nel passaggio dal dovere tributario - un impegno inderogabile di solidarietà economica, adempiuto dagli individui attraverso il trasferimento della ricchezza privata alle organizzazioni pubbliche alla consapevolezza del diritto tributario, vale a dire del diritto che ha ciascun consociato a vedere garantito il proprio benessere attraverso l'erogazione di servizi alla comunità (sanità, istruzione, sicurezza, difesa ecc.) finanziati mediante un prelievo equo e attuato secondo procedure giuste.

\footnotetext{
12 Ibid.

13 Parole pronunciate da Ezio Vanoni nel 1945 e riportate in A. Magliulo, Ezio Vanoni. La giustizia sociale nell'economia di mercato, Edizioni studium, Roma, 1991.
} 


\section{Bibliografia}

De Mita E., Maestri del diritto tributario, Giuffrè, Milano, 2013

Einaudi L., Miti e paradossi della giustizia tributaria, Einaudi, Torino, 1938

Forte F., Luigi Einaudi e il mercato e il buongoverno, Einaudi, Torino, 1982

Gallo F., Etica, fisco e diritti di proprietà, in Rass. trib., 2008, 11 ss.

Id., La funzione del tributo ovvero l'etica delle tasse, in Riv. trim. dir. pubbl., 2009, 399 ss.

Id., Disuguaglianze, giustizia distributiva e principio di progressività, in Rass. trib., 2012, 287 ss.

Id., L'evoluzione del sistema tributario e il principio di capacità contributiva, in Rass. trib., 2013, 499 ss.

Lupi R., Le illusioni fiscali, Il Mulino, Bologna, 1996

Id., Evasione fiscale, paradiso e inferno, Ipsoa, Milano, 2008

Magliulo A., Ezio Vanoni. La giustizia sociale nell'economia di mercato, Edizioni Studium, Roma, 1991

Marongiu G., Introduzione, in AA.VV., Lezioni di diritto tributario, IV ed., Giappichelli, Torino, 2013

Id., L'imposta personale e progressiva nel pensiero e nell'opera di Ezio Vanoni, in Dir. prat. trib., 2000, I, 511 ss.

Santoro A., L'evasione fiscale, Il Mulino, Bologna, 2010

Smith A., An Inquiry into the Nature and Causes of the Wealth of Nations, 1776, trad. it., Ricerche sopra la natura e le cause della ricchezza delle Nazioni, Mondadori, Milano, 1977

Tremonti G., Una nota di politica fiscale: la crisi dell'Irpef e la questione della progressività. Il caso Italia, in Riv. dir. fin. sc. fin., 1999, 3

Id., Le cause e gli effetti politici della prima crisi globale, in Riv. dir. fin. sc. fin., 2010, 3

Vanoni E., Opere giuridiche, vol. I, Giuffrè, Milano, 1961

Id., Opere giuridiche, vol. II, Giuffrè, Milano, 1962

Vigna G., Ezio Vanoni: il sogno della giustizia fiscale, Rusconi, Milano, 1993 


\section{STRUTTURE MATEMATICHE PER IL BENESSERE: IL TEOREMA DI ARROW}

Simone Camosso*

\section{Introduzione}

Con il termine benessere, letteralmente "stare bene" o "esistere bene", denotiamo uno stato personale che caratterizza la qualità della vita della persona. Tale definizione, troppo generica da rendere operativa, verrà sostituita con la nozione di benessere sociale, più familiare in campo economico. Alla nozione di benessere sociale assoceremo poi una funzione (o relazione) detta di benessere sociale e dipendente dalle utilità dei singoli individui. Esistono tantissime tipologie di funzioni di benessere sociale, la prima è stata coniata da A. Bergson nel 1938 e si presentava non unicamente come funzione, ma come funzionale (per intenderci funzione di funzioni) di funzioni di utilità dei singoli individui. E' chiaro che dal punto di vista filosofico il concetto di benessere sociale è strettamente correlato a quello di scelta, per tale ragione si parlerà anche di funzione di scelta associata tipicamente a problemi di votazione elettorali. Parleremo in seguito delle proprietà caratterizzanti queste funzioni di scelta e benessere sociale, richiamando brevemente alcuni risultati classici della microeconomia e dell'economia politica soffermandoci ad

\footnotetext{
* Dottorando in Matematica Pura e Applicata Dipartimento di Matematica e Applicazioni dell'Università degli Studi di MilanoBicocca
} 
analizzare più in dettaglio il risultato fondamentale di Arrow, che ha influenzato e influenza ancora recentemente la ricerca. Come è noto, il teorema di Arrow, detto anche teorema dell'impossibilità di scelta, afferma che, sotto ipotesi opportune, non può esistere una funzione di scelta sociale come realizzazione di un modello democratico di scelta. Con metodo, utilizzando nozioni di logica comune, analizzeremo la prova del teorema fornita da Amartya Sen ed in seguito, introducendo brevi nozioni di analisi booleana, vedremo la versione quantitativa dello stesso teorema.

\section{Teoremi di benessere sociale}

Una funzione di benessere sociale è una funzione $S W=F\left(u_{1}, \ldots, u_{n}\right)$ con $\mathrm{u}_{1}, \ldots, \mathrm{u}_{\mathrm{n}}$ le utilità, tale per cui viene soddisfatta la condizione di Pareto, vedi nota ${ }^{2}$, e la cosiddetta avversione alla disuguaglianza, in nota $^{3}$. Esempi sono la funzione utilitaristica $S W=u_{1+} \ldots+u_{n}$, la funzione Rawlsiana $\mathrm{SW}=\min \left\{\mathrm{u}_{1}, \ldots, \mathrm{u}_{\mathrm{n}}\right\}$ e la funzione di Cobb-Douglas . Ipotizziamo di avere una situazione di scambio molto semplice: Adamo ed Eva che si scambiano beni, ognuno per massimizzare la propria utilità. Tale situazione può essere rappresentata utilizzando la scatola di Edgeworth. Ai due vertici opposti della scatola troviamo Adamo ed Eva mentre dentro abbiamo le curve di indifferenza. Un esempio è illustrato in figura.

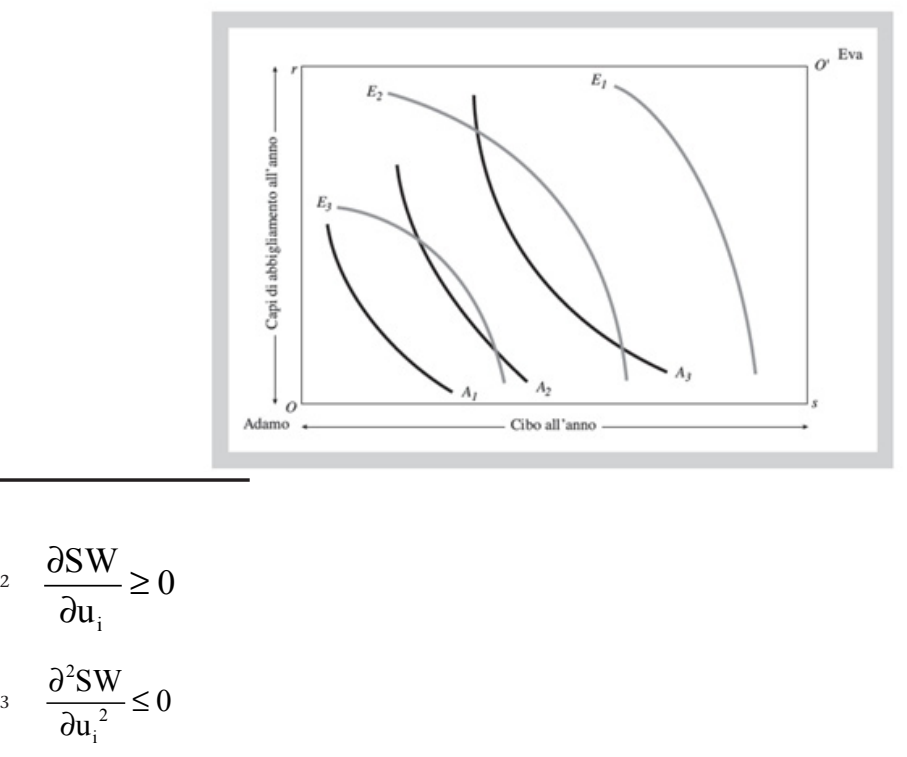


Questo è un esempio classico di come un problema economico può essere trasformato in un problema analitico geometrico. L'esistenza di un punto di equilibrio nel mercato può essere dedotto matematicamente da teoremi di punto fisso e dal punto di vista grafico, questi punti corrispondono ai punti delle due curve di indifferenza con uguale tangente. Unendo questi punti si ottiene la curva di contratto e si arriva alla formulazione dei primi due teoremi del benessere economico. Il primo teorema di benessere afferma che, in un sistema economico di libero mercato, con concorrenza perfetta e completezza dei mercati, le risorse vengono collocate in maniera pareto efficiente. A questo risultato di efficienza segue poi il secondo teorema del benessere che garantisce l'equità. Esso afferma, infatti, che l'allocazione Pareto ottimale può essere raggiunta da un'economia di libero scambio, a patto di ridistribuire appropriatamente le dotazioni iniziali.

\section{Gli studi empirici di Riker e il paradosso di Condorcet}

Nel 1982 William Riker ricostruì le preferenze dei 172 membri della casa dei rappresentanti su un voto del 1846 riguardante la riserva di Wilmot. L'oggetto della votazione riguardava l'appropriazione di 2.000.000 dollari per facilitare la negoziazione di un insediamento territoriale con il Messico alla fine della guerra, abolendo la schiavitù da tali territori. Le scelte erano tre: la scelta A prevedeva l'appropriazione del denaro senza la clausola sull'abolizione della schiavitù, la scelta B l'appropriazione del denaro con la clausola e la scelta $C$ in cui non si adotta nessuna azione per entrambe le cose. I risultati sono rappresentati nella tabella che segue.

\begin{tabular}{|l|l|l|}
\hline Gruppo politico & numero di voti & Preferenze \\
\hline Northern Administration Democrats & 7 & ABC \\
\hline Northern Free Soil Democrats & 51 & BAC \\
\hline Border Democrats & 8 & ABC 。 ACB \\
\hline Southern Democrats & 46 & ACB \\
\hline Northern Pro-war Whigs & 2 & CAB \\
\hline Northern Anti-war Whigs & 39 & CBA \\
\hline Border Whigs & 3 & BAC 。 BCA \\
\hline Southern and Border Whigs & 16 & ACB \\
\hline
\end{tabular}

Come è possibile osservare, abbiamo che A vince su C (120-41), C vince su B (103-58) e B vince su A (90-71) (per maggiori informazioni si veda D in Bibliografia). Tale situazione paradossale era già 
stata notata nel diciottesimo secolo dal Marchese Nicolas de Condorcet, che osservò come le preferenze collettive possono essere cicliche. Cerchiamo di tradurre questo in un linguaggio più formale. Indichiamo con $\mathrm{x}, \mathrm{y}, \mathrm{z}, \ldots$ i candidati ad una votazione e definiamo un ordine su tale insieme. Diremo che $\mathrm{x}>\mathrm{y}$ se e solo se il candidato $\mathrm{x}$ sconfigge il candidato $\mathrm{y}$. Ricordiamo che la relazione di ordine gode di tre proprietà: deve essere riflessiva $x>x$, antisimmetrica: se $\mathrm{x}>\mathrm{y}$ e $\mathrm{y}>\mathrm{x}$ allora $\mathrm{x}=\mathrm{y}$ e transitiva: se $\mathrm{x}>\mathrm{y}$ e $\mathrm{y}>\mathrm{z}$ allora $\mathrm{x}>\mathrm{z}$. Da queste elementari definizioni il paradosso di Condorcet può essere riformulato dicendo che le preferenze di maggioranza possono essere irrazionali (intransitive) anche quando le preferenze individuali sono razionali (transitive). In pratica si sta utilizzando la proprietà transitiva per esprimere un concetto di razionalità. Intransitività o irrazionalità, nel caso precedente, corrisponde all'avere ciclicità senza un vincitore.

\section{Gli assiomi di Arrow e il Teorema di Arrow}

Prima di enunciare il teorema di Arrow vediamo quali sono gli assiomi postulati da Kenneth Arrow. Li elenchiamo nel seguente modo: U (Non restrizione del dominio), per ogni insieme logicamente possibile di preferenze individuali c'è un ordine sociale $\mathrm{R}=$ z; I (Indipendenza dalle alternative irrilevanti), la classifica sociale di ogni paio $\{x, y\}$ dipenderà solamente sulle classifiche individuali di x e y; P (Principio di Pareto), se ognuno preferisce $\mathrm{x}$ a $\mathrm{y}$ allora $\mathrm{x}$ è socialmente preferito a y; D (Non dittatorialità), non c'è nessuna persona tale per cui, se questa preferisce $\mathrm{x}$ a $\mathrm{y}$, allora $\mathrm{x}$ è socialmente preferito a y, senza il parere degli altri. Assumendo la validità di tali assiomi enunciamo il teorema di Arrow nel modo seguente: se ci sono almeno tre distinti stati sociali e un numero finito di individui, allora nessuna funzione di benessere sociale può soddisfare U,I,D e P.

Prima di fornire una prova introduciamo alcune definizioni che saranno utili in seguito. Diremo che un insieme G di elettori è "localmente decisivo" su un qualche paio di alternative $\{x, y\}$ se $x$ è socialmente preferito a y. Analogamente diremo che un insieme G di elettori è "globalmente decisivo" se è localmente decisivo per ogni paio di candidati $\{\mathrm{x}, \mathrm{y}\}$. Dimostriamo ora un lemma detto anche lemma di "diffusione”. Esso recita nel seguente modo: se G è localmente decisivo su $\{x, y\}$ allora $G$ è globalmente decisivo. Infatti sia 
$\{a, b\} \neq\{x, y\}$ e supponiamo che per $G$ si ha $a>x, x>$ y e $y>b$ e che per gli altri a > x e y > b. Per P, a è socialmente preferito a $x$ e y è socialmente preferito a b. Per la decisività di $G$ si ha che x è socialmente preferito a y. Per transitività a è socialmente preferito a b. Per I il fatto è relativo per le preferenze individuali su $\{a, b\}$. Infine solo le preferenze di $G$ sono state specificate quindi $G$ è decisivo per $\{a, b\}$. Ora per l'arbitrarietà di $\{a, b\}$ segue il lemma. Prima di vedere la dimostrazione del teorema di Arrow abbiamo bisogno di un secondo lemma, detto, questa volta, lemma di "contrazione". Questo lemma afferma che se G è decisivo, allora qualche sottoinsieme proprio è decisivo allo stesso modo di G. Per dimostrarlo sia G dato dall'unione di $G^{1}, G^{2}$ tale per cui ognuno in $G^{1}$ preferisce $x>y$ e $x>z$ (gli altri non specificati) e ognuno in $G^{2}$ preferisce $x>y$ e $z>y$. Per ipotesi di decisionalità di $\mathrm{G}$ si ha che $\mathrm{x}>\mathrm{y}$ e ci sono due casi. Nel primo z è allo stesso livello di $\mathrm{x}$ allora $\mathrm{z}>\mathrm{y}$ e per la decisionalità di $\mathrm{G}^{2} \mathrm{su}\{\mathrm{z}, \mathrm{y}\}$ si ha che $\mathrm{G}^{2}$ è globalmente decisivo. Nel secondo z è allo stesso livello di $y$ allora $\mathrm{x}>\mathrm{z}$ e per la decisionalità di $\mathrm{G}^{1} \mathrm{su}\{\mathrm{z}, \mathrm{x}\}$ si ha che $\mathrm{G}^{1}$ è globalmente decisivo. Abbiamo tutti gli elementi necessari per la dimostrazione del teorema di Arrow. Infatti, per P applicato su tutti gli elettori abbiamo che tutti gli elettori sono decisivi. Per il lemma di contrazione qualche sottoinsieme proprio di questi elettori è decisivo ma, il lemma di contrazione può essere applicato tante volte fino a quando una persona sola risulta decisiva e questo viola D. Osserviamo, inoltre, come, togliendo l'assioma di non dittatorialità, sia effettivamente possibile trovare una funzione che soddisfi gli altri assiomi che è per l'appunto la funzione dittatoriale (per maggiori informazioni si veda A in Bibliografia).

\section{Formulazione quantitativa del Teorema di Arrow}

Consideriamo ora il caso di una votazione di tre votanti e la funzione $\operatorname{MAJ}_{3}(\mathrm{x})=\operatorname{sign}\left(\mathrm{x}_{1}+\mathrm{x}_{2}+\mathrm{x}_{3}\right)$ detta funzione maggioranza (dove sign è la funzione segno) e definita su $\{-1,+1\}^{3}$ in $\{-1,0,1\}$ (ricordiamo che in generale è possibile considerare il caso di $n$ votanti). Adesso osserviamo che la funzione precedente può essere scritta come $\operatorname{MAJ}_{3}(\mathrm{x})=\frac{1}{2} \mathrm{x}_{1}+\frac{1}{2} \mathrm{x}_{2}+\frac{1}{2} \mathrm{x}_{3}-\frac{1}{2} \mathrm{x}_{1} \mathrm{x}_{2} \mathrm{x}_{3}$. Quello che 
si è precedentemente utilizzato è un'espansione di Fourier di una funzione Booleana ( funzione con dominio +1 o -1 a valore reale). Data una funzione $\mathrm{f}:\{-1,+1\}^{\mathrm{n}} \rightarrow \square$ si definisce l'espansione di Fourier associata ad fla serie $\sum_{\mathrm{S} \subseteq[\mathrm{n}]} \hat{\mathrm{f}}(\mathrm{S}) \chi_{\mathrm{S}}(\mathrm{x})$ dove $\chi_{\mathrm{S}}(\mathrm{x})=\prod_{\mathrm{i} \in \mathrm{S}} \mathrm{x}_{\mathrm{i}}$ definita da $\{-1,1\}^{\mathrm{n}}$ a valori in $\{-1,+1\}$ è detta funzione parità che vale 1 se c'è un numero pari di - 1 nel vettore x e -1 altrimenti. Vediamo un esempio calcolando l' espansione di Fourier della funzione $\operatorname{MAJ}_{2}(\mathrm{x})=\operatorname{sign}\left(\mathrm{x}_{1}+\mathrm{x}_{2}\right)$. Il trucco è di utilizzare i polinomi multilineari (di Newton) (vedi nota ${ }^{*}$ ). Allo stesso modo si ricava l'espressione nel caso 3 dimensionale solo che in questo caso dovremo dividere per $2^{3}=8$ anziché 4 . Veniamo ora a qualche definizione. Un vettore $(\mathrm{a}, \mathrm{b}, \mathrm{c}) \in\{-1,+1\}^{3}$ è razionale se e solo se le componenti non sono tutte uguali. Data f una funzione booleana come sopra si definisce k-esimo peso di f e la stabilità del rumore come riportato nella nota ${ }^{* *}$, dove $\tilde{\mathrm{n}} \in[0,1]$. Definiamo la funzione NAE: $\{-1,+1\}^{3} \rightarrow\{1,0\}$ che restituisce 1 se e solo se le componenti del vettore $\mathrm{x}$ non sono tutti uguali, cioè se e solo se $\mathrm{x}$ è razionale. Proviamo ora un fatto importante che ci servirà in seguito (dovuto a Kalai) e che riguarda l'espansione in serie di Fourier della funzione NAE, tutto è riportato in nota $^{3}$. Vediamo ora in questo nuovo linguaggio come viene riformulato il teorema di Arrow. Definiamo f una funzione dittatoriale e la indichiamo con $\mathrm{f}= \pm$ DICT, una funzione tale che $\mathrm{f}(1,1,1)=1$ e $\mathrm{f}(-$ $1,-1,-1)=-1$. Enunciamo ora il teorema di Arrow: sia f una funzione che produce vettori razionali, se tutti i votanti sono razionali allora $\mathrm{f}= \pm$ DICT. ${ }^{4}$

4 troviamoun'espansionein serie diFourier della funzione NAE: $\{-1,+1\}^{3} \rightarrow\{1,0\}$ definita prima. Tale espansione risulta $\operatorname{NAE}(\mathrm{a}, \mathrm{b}, \mathrm{c})=\frac{3}{4}-\frac{1}{4}(\mathrm{ab}+\mathrm{bc}+\mathrm{ac})$
e si ha $\mathrm{E}[\mathrm{NAE}(\mathrm{f}(\mathrm{x}), \mathrm{f}(\mathrm{y}), \mathrm{f}(\mathrm{z}))]=\frac{3}{4}-\frac{3}{4} \operatorname{Stab}_{-1}(\mathrm{f})$ Infatti, poiché le variabili aleatorie $\mathrm{x}, \mathrm{y}$ e $\mathrm{y}, \mathrm{z}$ prese a coppie hanno la stessa distribuzione e per la linearità di E si ha $\operatorname{E}[\operatorname{NAE}(\mathrm{f}(\mathrm{x}), \mathrm{f}(\mathrm{y}), \mathrm{f}(\mathrm{z}))]$

$=\frac{3}{4}-\frac{1}{4}(\mathrm{E}[\mathrm{f}(\mathrm{x}) \mathrm{f}(\mathrm{y})]+\mathrm{E}[\mathrm{f}(\mathrm{y}) \mathrm{f}(\mathrm{z})]+\mathrm{E}[\mathrm{f}(\mathrm{x}) \mathrm{f}(\mathrm{z})])=\frac{3}{4}-\frac{3}{4} \mathrm{E}[\mathrm{f}(\mathrm{x}) \mathrm{f}(\mathrm{y})]$ ma $E\left[x_{i} y_{i}\right]=\frac{2}{6}(+1)+\frac{4}{6}(-1)=-\frac{1}{3} \operatorname{così~} E[f(x) f(y)]=\operatorname{Stab}_{-\frac{1}{3}}(f)$ 


\section{Dimostrazione del Teorema di Arrow (versione quan- titativa)}

La dimostrazione si articola come segue: supponiamo che la speranza di avere un vincitore di Condorcet sia 1 , cioè $\mathrm{E}[\operatorname{NAE}(\mathrm{f}(\mathrm{x}), \mathrm{f}(\mathrm{y}), \mathrm{f}(\mathrm{z}))]=1$ allora per la nota ${ }^{5}$ e la nota ${ }^{6}$, l'uguaglianza si

ha se e solo se $\mathrm{W}^{1}(\mathrm{f})=1$ cioè se e solo se $\mathrm{f}= \pm \chi_{\mathrm{S}}$ per qualche $|\mathrm{S}|=1$ cioè $\mathrm{f}= \pm$ DICT. Per maggiori dettagli matematici sulla dimostrazione si veda $B$ in Bibliografia.

\section{Conclusioni}

La versione quantitativa del Teorema di Arrow ci permette inoltre di avere stime quantitative su misure di probabilità riguardo a situazioni di votazioni elettorali. Ad esempio, se si considera una elezione, in cui il numero di votanti cresce tendendo all'infinito, la probabilità che la votazione sia "razionale" è circa 0,192 (o più precisamente vedi nota ${ }^{7}$ ). Di recente, oltre ai metodi dell'analisi booleana e ai metodi logici, si stanno sviluppando anche metodi geometrici per dimostrare questi tipi di risultati. E' il caso, ad esempio, di un recente Teorema di Sen nell'ambito della teoria delle scelte sociali, dove viene analizzata l'impossibilità di un "Libero Paretiano", ovvero dove viene dimostrato un conflitto tra il concetto di benessere standard e le diverse forme minimali di liberismo. La dimostrazione di tale risultato si fonda essenzialmente nella rappresentazione geometrica delle possibili classifiche su cubi. Ad esempio se si considerano tre alternative $\mathrm{A}, \mathrm{B}, \mathrm{C}$, e un ordinamento, allora è possibile associare ad ogni tripla di preferenze un vertice del cubo dove nel vertice $(0,0,0)$ e in $(1,1,1)$ vediamo chiaramente il paradosso di Condorcet.

$$
\begin{aligned}
& 5 \quad\left(-\frac{1}{3}\right)^{\mathrm{k}} \geq-\frac{1}{3} \text { per ogni } \mathrm{k} \\
& 6 \quad 1=\frac{3}{4}-\frac{3}{4} \mathrm{Stab}_{-\frac{1}{3}}(\mathrm{f})=\frac{3}{4}-\frac{3}{4} \sum_{\mathrm{k}=0}^{\mathrm{n}}\left(-\frac{1}{3}\right)^{\mathrm{k}} \mathrm{W}^{\mathrm{k}}(\mathrm{f}) \\
& 7 \quad \frac{3}{9}-\frac{3}{4}\left(1-\frac{2}{-} \operatorname{arcos}(-1 / 3)\right) \approx 0.192
\end{aligned}
$$




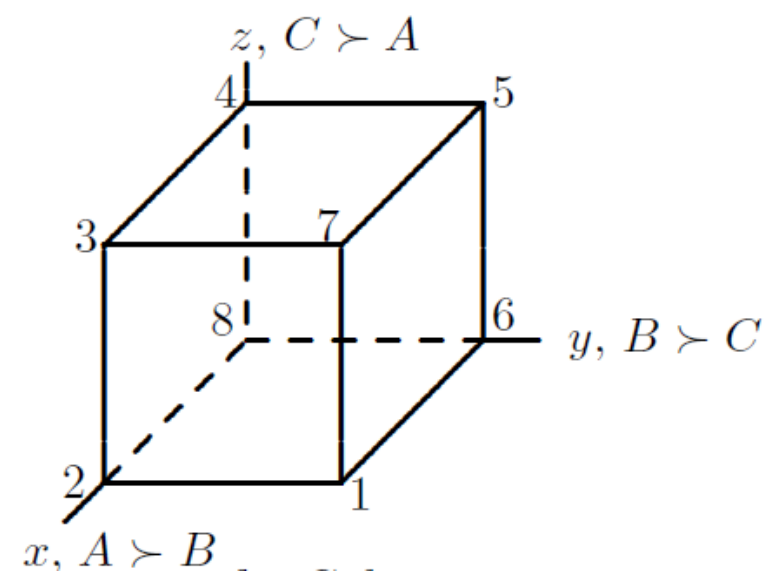

Il discorso viene poi generalizzato da Sen al caso di n alternative, utilizzando dei cubi n-dimensionali. Il risultato di impossibilità di Arrow sta al centro della moderna teoria della scelta sociale e nel corso di questi anni sono stati dati molti metodi di prova di questo risultato fondamentale. Citiamo come ultimo caso quello che utilizza un approccio topologico fornito da Baryshnikov del dipartimento di matematica della Osnabrück University, in Germania. L'approccio utilizzato da quest'ultimo è una rivisitazione del risultato ottenuto prima di Chichilnisky, dove però il risultato non era solo di tipo topologico. Al contrario, le funzioni di preferenza utilizzate erano lisce su uno spazio Euclideo e non banalmente lineari. Inoltre, le regole di aggregazione erano assunte continue in qualche topologia. Questa teoria sembra comunque avere più connessioni con la teoria dell'equilibrio, invece che con la classica teoria della scelta sociale. Diamo un'idea del lavoro svolto senza entrare nel dettaglio dell'argomentazione. In questo caso abbiamo come preferenze dei funzionali lineari definiti su un qualche spazio Euclideo di dimensione $\mathrm{n}$ aventi lunghezza 1.

Per quanto riguarda la regola di aggregazione, questa si presenta come una mappa f da uno spazio dei profili dato allo spazio delle preferenze che è una sfera con una dimensione in meno dello spazio Euclideo originario. Tale funzione deve essere continua e rispettare l'unanimità binaria, ovvero se una alternativa $\mathrm{x}$ è preferita ad una alternativa y da tutti i votanti, allora l'aggregato $\mathrm{f}(\mathrm{x})$ è maggiore di $\mathrm{f}(\mathrm{y})$. In questo contesto un manipolatore è un votante che da un vettore di preferenze qualsiasi utilizza unicamente la sua presenza per 
produrre un funzionale che rappresenti l'aggregato finale. Il teorema di Chichilnisky dice che se $\mathrm{n}>1$ (numero di votanti) allora esiste sempre un manipolatore. La dimostrazione del risultato si articola in due parti e richiede la conoscenza di strumenti matematici di topologia algebrica come il gruppo di omologia e i simplessi simpliciali. Nella prima parte si dimostra che, se il votante 1 è un manipolatore, allora l'operatore in Omologia (n-1) dimensionale associato ad $\mathrm{f}$ mappa l-esimo vettore base di $\mathrm{H}_{\mathrm{n}-1}$ (Profili) $=Z^{\mathrm{k}}$ nel generatore di $\mathrm{H}_{\mathrm{n}-1}($ Preferenze $)=\mathrm{Z}$ altrimenti in zero. Mentre la seconda parte è un semplice calcolo che prova che uno e soltanto uno dei vettori della base va nel generatore di $\mathrm{H}_{\mathrm{n}-1}$ (Preferenze) $=\mathrm{Z}$ e questo prova il teorema. Siamo arrivati alla fine del nostro percorso e, sperando di non essere stato troppo pedante, spero di avere reso l'idea dell'importanza del concetto di impossibilità che dal punto di vista filosofico interessa non solo i sistemi economici o sociali, ma molti altri campi della conoscenza.

$$
\begin{aligned}
& \text { * } \\
& \operatorname{MAJ}_{2}(\mathrm{x})=\frac{\left(1+\mathrm{x}_{1}\right)\left(1+\mathrm{x}_{2}\right)}{4} \mathrm{MAJ}_{2}(1,1)+\frac{\left(1+\mathrm{x}_{1}\right)\left(1-\mathrm{x}_{2}\right)}{4} \operatorname{MAJ}_{2}(1,-1)+\frac{\left(1-\mathrm{x}_{1}\right)\left(1+\mathrm{x}_{2}\right)}{4} \operatorname{MAJ}_{2}(-1,1)+ \\
& \frac{\left(1-\mathrm{x}_{1}\right)\left(1-\mathrm{x}_{2}\right)}{4} \mathrm{MAJ}_{2}(-1,-1)
\end{aligned}
$$

ora poiché $\operatorname{MAJ}_{2}(+1,-1)=\operatorname{MAJ}_{2}(-1,+1)=0$ rimangono solo il primo e l'ultimo termine, ottenendo così $\operatorname{MAJ}_{2}(\mathrm{x})=\frac{1}{2} \mathrm{x}_{1}+\frac{1}{2} \mathrm{x}_{2}$

${ }^{* *}$ Si definisce k-esimo peso di f e lo si indica come $\mathrm{Wk}(\mathrm{f})$ come $\sum_{|\mathrm{S}|=\mathrm{k}} \hat{\mathrm{f}}(\mathrm{S})^{2}$

e la stabilità del rumore come

$$
\operatorname{Stab}_{\rho}(\mathrm{f})=\sum_{\mathrm{S} \subseteq[\mathrm{n}]} \rho^{|\mathrm{S}|} \hat{\mathrm{f}}(\mathrm{S})^{2}=\sum_{\mathrm{k}=1}^{\mathrm{n}} \rho^{\mathrm{k}} \mathrm{W}^{\mathrm{k}}(\mathrm{f})=\mathrm{E}[\mathrm{f}(\mathrm{x}) \mathrm{f}(\mathrm{y})]
$$




\section{Bibliografia}

Arrow J.K., Arrow's theorem, Edizioni Steven N.Durlauf and Lawrence E. Blume, Palgrave Macmillan, 2008

Baryshnikov Y. M., Unifyng Impossibility Theorems: A Topological Approach, Editore: Advanced in applied Mathematics 14 (Università di Osnabrück), Germania, 1993

De Finetti B., Un matematico e l'economia, Milano, FrancoAngeli, 1969.

Gehrlein W.V., Condorcet's Paradox, Springer, Vienna, 2006

Li L. e Saari D.G., Social Choice and Welfare, Springer, Berlino, 2008

O'Donnel R., Some Topics in Analysis of Boolean Function, presentazione al 40th ACM STOC Conference, Canada, 2008

Sen A., The Impossibility of a Paretian Liberal, Editore: Journal of Political Economy $n^{\circ} 78$, Chicago, 1970 


\section{DIRITTO ALLA SALUTE, DISEGUAGLIANZE E MATERIALITÃ DEL SOCIALE: UNA RICOGNIZIONE PER LEGGERE LE MOLTEPLICI CORRELAZIONI TRA DIRITTO E SOCIETÂ NELL'AREA DEL BENESSERE}

\section{Carlo Botrugno*}

\section{Un diritto alla salute soltanto sulla Carta?}

Alla riflessione sociologico-giuridica va attribuito il merito di aver fatto emergere la distanza tra law in action e law in the books, ovvero di aver messo in luce quel particolare divario che separa la prescrizione normativa così come la si trova «sulla carta», dagli effetti desiderati o indesiderati che essa consegue nella praxis, ovvero nella sua «vita giuridica» concreta. L'indagine sull'efficacia del diritto ha contribuito a rovesciare l'approccio tradizionale delle scienze giuridiche secondo il quale una norma si considera «efficace» allorché sia «oggettivamente in grado di esplicare i propri effetti». Dalla distinzione tra validità ed efficacia della norma giuridica discende la rilevanza assunta dall'enforcement, ovvero da quel complesso di attività normative finalizzate a porre in essere le condizioni concrete, sia giuridiche, sia economiche, necessarie a garantire una concreta efficacia al dettato normativo ${ }^{1}$.

\footnotetext{
* Dottorando in Diritto e Nuove Tecnologie Centro Interdipartimentale di Ricerca di Storia del diritto, Filosofia e Sociologia del diritto e Informatica giuridica dell’Università di Bologna

1 V. Ferrari, Lineamenti di Sociologia del diritto, Laterza, Roma/Bari, 2004, pp. 261 e ss.
} 
L'ordinamento giuridico italiano faceva esperienza di tale necessità già a partire dall'entrata in vigore della Costituzione repubblicana, e, in particolare, seppur non in via esclusiva, con riferimento alla garanzia dei c.d. diritti sociali, ovvero di quelle posizioni giuridiche di vantaggio attribuite ai singoli per l'ottenimento di una prestazione positiva erogata dall'apparato pubblico ${ }^{2}$.

Una posizione di preminenza all'interno del dibattito che ha appassionato la scienza giuridica al fine di stabilire la natura immediatamente precettiva o, per contro, programmatica, delle disposizioni costituzionali, è stata assunta dal diritto alla salute codificato all'art. 32. Tale fattispecie infatti aveva il pregio di offrirsi agli interpreti e alla dottrina nella duplice veste di diritto fondamentale (ovvero di libertà, nella misura in cui stabiliva un obbligo di astensione dal porre in essere attività o dal compiere azioni che potessero rivelarsi lesive del diritto alla salute), e di diritto sociale (ovvero, a prestazioni positive, nella misura in cui riconosceva al singolo una facoltà di rivendicare una pretesa diretta al conseguimento di una prestazione di tutela della salute).

Il diritto sociale alla salute - al quale si farà riferimento d'ora innanzi ai fini di questa riflessione - vanta nel contesto attuale una piena dignità di diritto costituzionale ${ }^{3}$, seppure il suo godimento effettivo risulti condizionato dalla contingente attuazione per opera del legislatore ordinario, e conseguentemente, dalla necessaria ponderazione di questo con ulteriori interessi protetti a livello costituzionale ${ }^{4}$.

Nondimeno, per offrire una ricognizione in chiave critica sull'effettività del diritto alla salute, è opportuno abbandonare il «cielo dei concetti giuridici», tentando così di conciliare l'utilizzo delle categorie sociologico-giuridiche con l'analisi dei fattori sociali, cultura-

\footnotetext{
2 Per un inquadramento teorico della categoria dei diritti sociali in relazione ai diritti fondamentali si veda N. Bobbio, L'età dei diritti, Einaudi, Torino, 2008 [1990]; L. Ferrajoli, (a cura di E. Vitale) Diritti fondamentali: un dibattito teorico, Laterza, Roma/ Bari, 2001.

3 Su questo punto appaiono convincenti le considerazioni di E. Cavasino, La flessibilità del diritto alla salute, 2012, Editoriale Scientifica, Napoli, p. 7, che in accordo con la dottrina oggi prevalente, rigetta la tesi dell'inferiorità dei diritti sociali, ovvero del rapporto di subordinazione di questi ultimi rispetto ad altri principi fondamentali della Costituzione.

${ }_{4}$ Imprescindibile in questo contesto è il riferimento alla sentenza n. 455/1990 che cristallizza le precedenti elaborazioni della Corte Costituzionale in materia. Secondo E. Cavasino, cit., pp. 91 e ss., vi è consenso ampio sulla triplice natura condizionata del diritto alle prestazioni di salute: disponibilità finanziaria, possibilità tecnica, ed attuazione legislativa. Per quanto concerne il vincolo finanziario ulteriori considerazioni sono contenute all'interno di questa riflessione.
} 
li, ed economici, che sovente inibiscono una piena fruibilità delle posizioni di vantaggio dei singoli. Il diritto alla salute verrà pertanto indagato mettendo in evidenza le correlazioni che possono essere individuate tra il diritto e la società, e che appaiono in grado di offrire una comprensione dei meccanismi attraverso i quali la diversificazione dello stato di salute e della sua protezione giuridica si producono attraverso dinamiche di carattere circolare tra la materialità del sociale e le opzioni politico-organizzative che ispirano i contemporanei sistemi sanitari dei paesi industrializzati.

\section{Le correlazioni che incidono sulle diseguaglianze in salute: dal diritto alla società...}

Le proclamazioni formali dell'uguaglianza all'interno delle costituzioni rigide del XX secolo hanno indotto per lungo tempo a porre in secondo piano l'indagine sulle modalità attraverso le quali il diritto rectius l'organizzazione sanitaria potesse determinare discriminazioni a carattere latente nella garanzia del diritto alla salute ${ }^{5}$.

Il collasso della finanza pubblica e la graduale, persistente, carenza di risorse che ha travolto gli equilibri derivanti dai c.d. trenta gloriosi, ha costretto tuttavia i sistemi sanitari dei paesi industrializzati a rimodulare la declinazione dei principi di universalismo, tanto che a esso è venuto ad affiancarsi stabilmente l'attributo della 'selettività'. L'ossimoro incarnato dalla nozione di universalismo selettivo può rappresentare efficacemente il livello di protezione attuale del diritto alla salute, la cui natura finanziariamente condizionata è emersa come un dato di fatto allorché anche la giurisprudenza costituzionale ha consolidato un orientamento in base al quale il diritto del singolo a ricevere una prestazione di cura, per quanto fondamentale, non potesse considerarsi assoluto (ovvero da garantire a prescindere da ogni altra valutazione di carattere finanziario), e che pertanto potesse essere compresso a salvaguardia di ulteriori interessi costituzionalmente protetti, tra cui, segnatamente il diritto/dovere dello Stato alla più efficiente allocazione possibile delle risorse pubbliche ${ }^{6}$. È altresì noto che, in base a tale orientamento,

\footnotetext{
5 Il fenomeno è stato oggetto di recente attenzione anche da parte dell'Agenzia Europea per i Diritti Umani (FRA). Cfr. la Relazione Eu-Midis Data: Multiple discrimination, reperibile su: fra.europa.eu/fraWebsite/research/publications/publications_per_year/2011/pub-multiple-discrimination_en.htm.

6 A questo proposito è d'obbligo il riferimento al dibattito sorto attorno alla fat-
} 
il bilanciamento fra gli opposti interessi non potesse in nessun caso spingersi sino a provocare una compromissione del livello essenziale della protezione giuridica della salute, ovvero di quella parte che ne realizza il nucleo irriducibile ${ }^{7}$. Il giudice costituzionale mutuava in questo proposito la nozione di Wesensgehaltsgarantie ${ }^{8}$, che ha trovato ampia corrispondenza nell'ambito delle fonti internazionali del diritto alla salute con il riferimento di core obligation. Tale nozione rimanda ad un nucleo di tutela inderogabile in virtù del fatto che questa appare realizzabile attraverso un dispendio di risorse così «infimo» da non poter essere distratto al fine di realizzare migliori allocazioni alternative ${ }^{9}$.

Se pertanto lo Stato può legittimamente predisporre un'organizzazione di carattere selettivo-allocativa nell'area della sanità pubblica, nondimeno le modalità concrete assunte dalla stessa appaiono in grado di generare una diversificazione de iure nell'accesso alle prestazioni di tutela della salute, generando pertanto diseguaglianze in salute politicamente determinate.

Per quanto concerne il sistema sanitario italiano, molteplici stagioni di riforme ispirate ai principi del decentramento amministrativo e dell'aziendalizzazione delle unità ospedaliere hanno inciso in maniera indelebile sull'omogeneità dei servizi offerti, nonostante l'ordinamento giuridico abbia tentato di scongiurare l'emersione di difformità territoriali attraverso l'istituzione dei livelli (prima uniformi, poi essenziali) delle prestazioni ${ }^{10}$. I livelli essenziali sono stati in seguito costituzionalizzati attraverso la riforma del Titolo V del 2001 che li ha resi il perno della garanzia «delle prestazioni concernenti i diritti civili e sociali che devono essere garantiti su tutto il

tispecie dei diritti ad "attuazione progressiva”, derivanti dal dettato dell'art. 2 del Patto Internazionale sui diritti economici, sociali e culturali del 1966. Per un approfondimento sul punto si rinvia al General Comment n.3/1990 del Comitato sui diritti economici, sociali e culturali, The nature of States parties 'obligations (V sessione, 1990), U.N. Doc. E/1991/23, annex III, 86 (1991).

7 Cfr. in proposito Corte Cost. n. 282/2002.

8 Così E. Cavasino, cit., p. 102.

9 Quanto risulta specificamente dal General Comment n. 14/2000 del Comitato per i Diritti Economici, Sociali e Culturali, E/C.12/2004/4, relativo all'art. 12 del Patto del 1966 sui diritti economici, sociali e culturali, e nello specifico al par. 48.

10 I Livelli Essenziali di Assistenza (LEA) previsti dal D. Lgs n. 502/1992. i livelli nel settore della sanità verranno definiti con D.P.C.M. del 29/11/2001. Su di un piano distinto ma correlato al processo di regionalizzazione del SSN devono essere menzionati inoltre i rapporti di convenzione tra enti pubblici e privati, le cui concrete modalità di incontro possono determinare differenze nell'offerta di servizi e nelle possibilità di accesso agli stessi. Così M. Tognetti Bordogna, Diseguaglianze di salute e immigrazione, Franco Angeli, Milano, 2008, p. 12. 
territorio nazionale ${ }^{11}$ in un sistema che, rovesciando il precedente riparto delle competenze, ha attribuito alle Regioni la gestione diretta delle attività di tutela della salute.

A partire dalla riforma costituzionale pertanto, ha preso avvio la c.d. regionalizzazione del Servizio Sanitario Nazionale, che se da una parte ha inteso avvicinare la gestione delle risorse pubbliche al governo locale ${ }^{12}$, dall'altra viene in rilievo come un processo in grado di accrescere la distanza tra i vari modelli organizzativi, e, quindi, almeno potenzialmente, di provocare un aggravio delle diseguaglianze territoriali in salute ${ }^{13}$. A questo proposito emerge in maniera sempre più netta un divario geografico a sfavore delle Regioni meridionali del paese che assume le forme di una inadeguatezza nell'offerta dei servizi (dall'assistenza ospedaliera ai programmi di screening oncologici, sino alla gestione della salute mentale, ecc.) ${ }^{14}$. Inoltre, pur senza poter stabilire in questa sede un nesso inferenziale tra qualità delle prestazioni e aspettative individuali, non si può tralasciare il fatto che a tale divario corrisponde una percezione diffusa secondo cui la qualità delle cure offerte nelle Regioni meridionali è inferiore al resto del paese, motivo per il quale i cittadini-pazienti continuano ad alimentare in maniera consistente fenomeni di mobilità interregionale, anche noti come migrazioni sanitarie ${ }^{15}$.

11 Così l'art. 117. co., 2, lett. $m$ ) come modificato dalla L. Cost. n. 3/2001.

12 Si veda la legge delega n. 42/2009 ed i conseguenti decreti attuativi, tra cui in particolare, il D. Lgs. n. 68/2011.

13 A questo proposito tuttavia si veda Lucchini M., Sarti S., Tognetti Bordogna M., I Welfare Regionali e le differenze territoriali nelle disuguaglianze di salute, in Schizzerotto A., Saraceno C., Brandolini A., (a cura di) Dimensioni della disuguaglianza in Italia: povertà, salute, abitazione, Il Mulino, Bologna, 2009, i quali ritengono che la portata delle diseguaglianze geografiche sia minore rispetto a quella determinata dalle condizioni sociali. Inoltre si rinvia all'esame dei rapporti annuali di OsservaSalute che documentano le differenze nell'erogazione delle prestazioni di cura sul territorio nazionale.

14 Così G. Costa, Diseguaglianze nella salute Nord-Sud Italia, in Salute Internazionale, 21/11/2011 http://www.saluteinternazionale.info/2011/11/diseguaglianze-nellasalute-nord-sud-in-italia/.

15 Anche in questo caso sono le regioni meridionali ed insulari le più colpite dal fenomeno. Per un approfondimento si rinvia a C. Zocchetti, La mobilità sanitaria tra regioni: Quanto, Dove, Per fare cosa?, in Statistica \& Società, anno 1, n. 2. A questo proposito sono illuminanti i dati che confermano un bilancio ampiamente negativo nel rapporto tra "pazienti in entrata" e "pazienti in uscita" nelle regioni meridionali. Nel 2011 questo bilancio è di segno negativo per la Sicilia (-38.055), per la Calabria (-52.800), per la Puglia (-34.909), per la Campania (-59.854). La regione settentrionale che presenta il valore negativo più alto è il Piemonte (-6.909). Particolarmente rilevante inoltre il dato dell'Emilia Romagna (+71.886). Fonte Il Sole-24 Ore Sanità su dati Sdo e Regioni, consultabile su http://www.sanita.ilsole24ore.com/art/regioni-eaziende/2013-04-17/mobilita-sanitaria-esodo-cerca-112745.php?uuid=AbhEx0nH. 


\section{... e dalla società al diritto}

Su di un piano distinto si colloca l'analisi delle variabili de facto (personali, sociali, culturali, economiche), e quindi esterne ai sistemi sanitari, che incidono sull'effettività della protezione della salute. La riflessione e la conoscenza relative all'azione di questi fattori si devono all'impegno profuso dalla Commission on Social Determinants of Health (CSDH) presso l'Organizzazione Mondiale della Sanità, le cui ricerche hanno permesso di svincolare la consistenza e il volume delle diseguaglianze in salute dai livelli di spesa sanitaria complessivi, nonché dal raggiungimento di performance di eccellenza nell'ambito della sanità pubblica ${ }^{16}$.

Tali fattori devono essere considerati in base al diverso condizionamento che esercitano in negativo sulla tutela della salute. Se da una parte, infatti, vengono in rilievo variabili quali il livello di istruzione, il genere, le condizioni socio-economiche, e la persistenza di fattori di discriminazione, che producono un effetto diretto sui livelli di accessibilità e di fruizione dei servizi di cura ${ }^{17}$, dall'altra parte bisogna considerare presupposti quali le condizioni lavorative e abitative, gli stili di vita, le abitudini alimentari, che appaiono produrre una distribuzione epidemiologica socialmente determinata ${ }^{18}$, ovvero correlata a quel complesso di condizioni che formano il contesto vi-

\footnotetext{
16 A questo proposito si rinvia alla lettura del Rapporto prodotto dalla Commissione, Social determinants of health The Solid Facts, giunto alla sua II edizione: www.euro. who.int/_data/assets/pdf.../e81384.pdf.

17 Cfr. A questo proposito si veda in particolare M. Geddes di Filicaia, Le diseguaglianze nell'assistenza sanitaria, in Osservatorio Italiano sulla salute globale. A caro prezzo, ETS, Pisa, 2006; G. Domenighetti, e J. Maggi, Definizione di priorità sanitarie e razionamento: efficacia ed efficienza verso equità e compassione, in Tendenze Nuove, marzo-aprile 2001; E. Reale, (a cura di ), Una salute a misura di donna, in Atti del gruppo di lavoro 1999-2001 "Medicina donna salute", Dip.to Pari Opportunità, Pres. Consiglio Ministri, Roma, 2002; S. Sarti, La classe sociale in buona salute, in Polis, 2006, n. 3. 18 Sulle diseguaglianze di salute tra Sud e Nord del paese si veda G. Padovani, Il diritto negato. La salute e le cure sono uguali per tutti?, Il Pensiero Scientifico, Roma, 2008, pp. 10 e ss., che documenta lo svantaggio del meridione, ovvero il fatto che nelle regioni del sud, isole comprese, ci si ammala di più. Per la relazione tra mortalità e classe sociale si veda: J. Fox, Health inequalities in European countries, Gower Press, Aldershot, 1989; A. E. Kunst, e J. P., Mackenback, An International comparison of socioeconomic inequalities in mortality, Department of Public Health and Social Medicine, Erasmus University Rotterdam, 1992. Per la relazione tra mortalità e grado di istruzione si veda S. V. Subramanian, P. Belli, e I. Kawachi, The macroeconomic determinants of health, in Annual Review of Public Health, 2002, n. 23; I. Kawachi, Social capital and community effects on population and individual health, in Annals of the New York Academy of Sciences, 1999, n. 896; G. Costa, e F. Faggiano, (a cura di), L'equità nella salute in Italia. Rapporto sulle diseguaglianze sociali in sanità, Franco Angeli, Milano, 1994.
} 
tale del soggetto - in senso fenomenologico il suo essere al mondo.

Nell'indagine relativa all'incidenza di questi fattori una posizione di rilievo è assunta dalla condizione migrante, che si rivela di fatto una causa di vulnerabilità di carattere multidimensionale ${ }^{19}$, e che appare strettamente correlata a fenomeni di discriminazione etnica/razziale, fattore la cui gravità è tale da condizionare più di ogni altro le possibilità concrete di tutela della salute da parte dei cittadini stranieri ${ }^{20}$. A questo proposito non possono essere sottaciute le difficoltà che i migranti affrontano nei paesi dell'Unione europea per guadagnare l'accesso ad una posizione giuridica regolare ${ }^{21}$, il cui ottenimento appare in misura sempre maggiore come necessario per un accesso pieno alle prerogative di cui è composto il diritto alla salute. In quest'ambito il caso più eclatante di diversificazione della tutela riguarda senza dubbio la Spagna, che con l'emanazione del Real Decreto Ley n. 16/2012 ha escluso gli immigrati in condizione irregolare dalla fruizione delle cure «non urgenti» ${ }^{22}$ infliggendo un duro colpo all'universalismo del sistema sanitario spagnolo, e tracciando un discrimine manifesto rispetto alle reali possibilità di cura dei cittadini stranieri privi di un regolare titolo di soggiorno.

\section{Conclusioni: diritto alla salute è giustizia?}

L'analisi delle diseguaglianze in salute costituisce il punto di avvio di una riflessione che assume sovente i contorni di una questione

\footnotetext{
19 Una vasta messe di studi ha messo in evidenza il peso della condizione di migrante sulla tutela della salute In particolare E. Reyneri, Sociologia del mercato del lavoro. Vol. II, Le forme dell'occupazione, Il Mulino, Bologna, 2011, documenta come le donne immigrate siano più esposte a traumi, incidenti, e malattie professionali rispetto alle donne italiane, nonostante il maggior livello di occupazione delle ultime. Inoltre, M. D., Hayward, M. Heron, Racial inequality in active life among older Americans, in Demography, 1999, n. 36; G. Costa, M. Cardano, e M. Demaria, Torino, storie di salute in una grande città. Città di Torino, Ufficio di statistica, Osservatorio Socioeconomico Torinese, 1998. M. Tognetti Bordogna, Nuove diseguaglianze in salute: il caso degli immigrati, in Cambio, III, 5, 2013.

20 A questo proposito, in aggiunta ai riferimenti già citati, si rinvia a M. Marceca, S. Geraci, M. Ardirò, Esperienza migratoria, salute e diseguaglianze, in Osservatorio Italiano sulla salute globale, cit., 2006.

${ }_{21}$ Per una trattazione della normativa in materia di immigrazione all'interno dell'Unione europea sia consentito fare rinvio a C. Botrugno, Immigrazione e Unione europea: un excursus storico ragionato, in Sociologia del diritto, n. 1, 2014.

22 Per un commento alla riforma si veda Controriforma sanitaria in Spagna. Nel mirino anche gli immigrati, in Salute Internazionale, su http://www.saluteinternazionale. info/2012/10/controriforma-sanitaria-in-spagna-nel-mirino-anche-gli-immigrati/.
} 
di giustizia, divenendo pertanto terreno d'elezione per il dibattito filosofico-giuridico che ha contribuito progressivamente a ricondurre il tema della corretta allocazione delle risorse in sanità ai principi di equità distributiva ${ }^{23}$. Il concetto di 'disuguaglianza' infatti incorpora un elemento morale e normativo idoneo a distinguerlo dal termine 'differenza', che può invece considerarsi come meramente descrittivo. In quest'ambito vi è ampio consenso sull'idea che, nella misura in cui appaiano evitabili, le diseguaglianze in salute siano anche ingiuste, e pertanto l'obiettivo di ridurle assurge ad imperativo di natura etica ${ }^{24}$. Pur non potendo dare conto in maniera esaustiva dell'ampiezza di tale dibattito non si può fare a meno di ricordare sinteticamente la visione di Norman Daniels, il quale, rifacendosi alla Theory of Justice di John Rawls, e in particolare, al principio di differenza, utilizza l'approccio dell'uguaglianza di opportunità al fine precipuo di definire una Just Health Care. Secondo Daniels, la protezione della salute è essenziale per il mantenimento dei normal functionings, intesi alla stregua di presupposti fondamentali per una piena partecipazione alla vita sociale e politica da parte degli individui $^{25}$. Nella sua elaborazione, nondimeno, Daniels prende le distanze dalla formulazione originaria di Rawls, che escludeva esplicitamente infortuni e patologie dai fattori da tenere in considerazione ai fini di una redistribuzione delle risorse ${ }^{26}$.

Per converso, le teorie utilitaristiche ispirano un orientamento che, applicato nell'area della sanità pubblica, persegue un'allocazione delle risorse economiche che rappresenti il miglior risultato possibile - the best outcome - ignorando differenze di natura relativa e relazionali tra gli individui. Dalla logica consequenzialista propria dell'utilitarismo deriva la rilevanza assunta dalla verifica dell'efficacia delle politiche sanitari, per la cui misurazione si suole ricorrere

\footnotetext{
23 Sulla connessione tra i principi di equità e di giustizia si veda A. Sen, On the status of equality, in Political theory, Vol. 24, n. 3, 1996. Per una rivisitazione del dibattito filosofico giuridico sul tema si rinvia a R. Mordacci, Una introduzione alle teorie morali. Confronto con la bioetica, Feltrinelli, Milano, 2003. Su di un piano distinto si veda inoltre E. Somaini, L'uguaglianza. Teorie, politiche, problemi. Donzelli, Roma, 2002.

${ }_{24}$ Così G. Berlinguer, nel parere elaborato dal Comitato Nazionale di Bioetica (CNB), Orientamenti bioetici per l'equità in salute, del 25/05/2001. L'obiettivo di ridurre le disuguaglianze sociali nell'ambito del diritto alla salute era già definito dalla Carta di Ottawa, approvata nell'ambito del Congresso Internazionale sulla Promozione della Salute tenutosi nell'omonima città canadese dal 17 al 21 novembre 1986. 25 Cfr. N. Daniels, Justice, Health and Healthcare, in The American Journal of Bioethics, Vol. 1, n. 2, 2001; N. Daniels., Just Health Care, Cambridge University Press, Cambridge, 1985.

26 J. Rawls J., Political Liberalism, Columbia University Press, New York, 1993, p. 194.
} 
ad indici quali il Quality-Adjusted-Life-Years (QUALs), e il DisabilityAdjusted-Life-Years (DALYs) ${ }^{27}$. Con riferimento a tali indicatori non si può trascurare l'analisi relativa all'incidenza del fattore reddito sulle opportunità di tutela della salute, e, in particolare, sulla modificazione delle aspettative di vita media alla nascita, e delle aspettative di vita media priva di malattie invalidanti. Se appare scontato, ictu oculi, sostenere che i livelli di reddito possano determinare maggiori o minori possibilità di tutela della propria salute - a questo proposito è sufficiente considerare tempestività ed appropriatezza - nondimeno, nella riflessione relativa al rapporto tra diseguaglianze in salute ed equità, si è cristallizzato un ampio consenso sulla particolare declinazione che questo fattore assume all'interno dei paesi industrializzati ${ }^{28}$. All'interno di tali paesi, più che il livello di reddito in sé, è la sua iniqua distribuzione - intesa come forbice di differenza tra il quintile più ricco e quello più povero della popolazione - a determinare una riduzione significativa nell'aspettativa di vita e di vita «in salute». In questi contesti emerge pertanto come la rigidità della stratificazione sociale sia idonea a provocare un peggioramento dello stato di salute ed una menomazione della relativa protezione giuridica.

In conclusione, si è inteso sottolineare come i determinanti politici delle diseguaglianze spesso rafforzino la condizione di vulnerabilità sociale che scaturisce dall'azione dei determinanti sociali della salute, dando origine ad una spirale patogenica di carattere multidimensionale che si autoalimenta (...stato di vulnerabilità à riduzione di capacità à stato di vulnerabilità...), ed a partire dalla quale emergono i tratti di quella che può essere definita come materialità del sociale ${ }^{29}$, una dimensione in cui la differenza si costituisce come ostacolo concreto rispetto alla protezione giuridica ed al godimento effettivo di beni primari, quali la salute e la vita stessa.

\footnotetext{
27 Così R. Rhodes, Justice in Medicine and Public Health, in Cambridge Quarterly of Healthcare Ethics, 2005, n. 14, p. 15.

28 Cfr. in particolare R. Wilkinson e K. Pickett The Spirit Level. Why More Equal Societies Almost Always Do Better, Allen Lane, London, 2009.

29 Il concetto è mutuato da P. Farmer, An anthropology of structural violence, in Current Anthropology, n. 3, vol. XLV, 2004.
} 


\section{Bibliografia}

Bobbio N., L'età dei diritti, Einaudi, Torino, 2008 [1990].

Botrugno C., Immigrazione e Unione europea: un excursus storico ragionato, in Sociologia del diritto, n. 1, 2014, 143-165.

Cavasino E., La flessibilità del diritto alla salute, Editoriale Scientifica, Napoli, 2012.

Costa G., e Faggiano F., (a cura di), L'equità nella salute in Italia. Rapporto sulle diseguaglianze sociali in sanità, Franco Angeli, Milano, 1994.

Costa G., Cardano M., Demaria M., Torino, storie di salute in una grande città, Osservatorio Socioeconomico Torinese, Ufficio di statistica, Città di Torino, 1998.

Costa G., Diseguaglianze nella salute Nord-Sud Italia, in Salute Internazionale, 21/11/2011 http://www.saluteinternazionale.info/2011/11/ diseguaglianze-nella-salute-nord-sud-in-italia/.

Daniels N., Just Health Care, Cambridge University Press, Cambridge, 1985.

Daniels N., Justice, Health and Healthcare, in The American Journal of Bioethics, Vol. 1, n. 2, 2001, 2-16.

Domenighetti G., e Maggi J., Definizione di priorità sanitarie e razionamento: efficacia ed efficienza verso equità e compassione, in Tendenze Nuove, marzo-aprile 2001, 4-14.

Farmer P., An anthropology of structural violence, in Current Anthropology, n. 3, vol. XLV, 2004, 305-325.

Ferrajoli L., (a cura di Vitale E.), Diritti fondamentali: un dibattito teorico, Laterza, Roma/Bari 2001.

Ferrari V., Lineamenti di Sociologia del Diritto, Laterza, Roma/Bari, 2004.

Fox J., Health inequalities in European countries, Gower Press, Aldershot, 1989.

Geddes di Filicaia M., Le diseguaglianze nell'assistenza sanitaria, in Osservatorio Italiano sulla salute globale. A caro prezzo, ETS, Pisa, 2006.

Hayward M. D., e Heron M., Racial inequality in active life among older Americans, in Demography, 1999, n. 36, 77-91.

Kawachi I., Social capital and community effects on population and individual health, in Annals of the New York Academy of Sciences, 1999, n. 896, 120-130.

Kunst A. E., e Mackenback J. P., An International comparison of socioeconomic inequalities in mortality, Department of Public Health and 
Social Medicine, Erasmus University Rotterdam, 1992.

Lucchini M., Sarti S., Tognetti Bordogna M., I Welfare Regionali e le differenze territoriali nelle disuguaglianze di salute, in Schizzerotto A., Saraceno C., Brandolini A., (a cura di) Dimensioni della disuguaglianza in Italia: povertà, salute, abitazione, Il Mulino, Bologna, 2009.

Marceca M., Geraci S., Ardirò M., Esperienza migratoria, salute e diseguaglianze, in Osservatorio Italiano sulla salute globale, ETS, Pisa, 2006.

Mordacci R., Una introduzione alle teorie morali. Confronto con la bioetica, Feltrinelli, Milano, 2003.

Padovani G., Il diritto negato. La salute e le cure sono uguali per tutti?, Il Pensiero Scientifico, Roma, 2008.

Rawls J., Political Liberalism, Columbia University Press, New York, 1993.

Reale E., (a cura di), Una salute a misura di donna, in Atti del Gruppo di lavoro Medicina Donna Salute 1999-2001, Dip.to. Pari Opportunità, Pres. Consiglio dei Ministri, Roma, 2002.

Reyneri E., Sociologia del mercato del lavoro. Vol. II, Le forme dell'occupazione, Il Mulino, Bologna, 2011.

Rhodes R., Justice in Medicine and Public Health, in Cambridge Quarterly of Healthcare Ethics, 2005, n. 14, 13-26.

Sarti S., La classe sociale in buona salute, in Polis, 2006, n. 3, 317-346.

Sen A., On the status of equality, in Political Theory, Vol. 24, n. 3, 1996, 394-400.

Subramanian S. V., Belli P., Kawachi I., The macroeconomic determinants of health, in Annual Review of Public Health, 2002, 23, 287-232.

Somaini E., L'uguaglianza. Teorie, politiche, problemi. Donzelli, Roma, 2002.

Tognetti Bordogna M., Diseguaglianze di salute e immigrazione, Franco Angeli, Milano, 2008.

Tognetti Bordogna M., Nuove diseguaglianze in salute: il caso degli immigrati, in Cambio, III, 5, 2013, 59-72.

Wilkinson R., e Pickett K., The Spirit Level. Why More Equal Societies Almost Always Do Better, Allen Lane, London, 2009.

Zocchetti C., La mobilità sanitaria tra regioni: Quanto, Dove, Per fare cosa?, in Statistica \& Società, anno 1, n. 2, 27-29. 


\title{
MUTILAZIONI GENITALI FEMMINILI: IL CONFLITTO TRA DIRITTI FONDAMENTALI E TRADIZIONI CULTURALI
}

\author{
MarianNa NobiLE*
}

\section{Premessa}

L'attuale configurazione multietnica e multiculturale della società occidentale impone che ci si interroghi sulle diverse modulazioni della relazione di cura e sulle richieste avanzate da individui appartenenti a comunità, le cui tradizioni spesso risultano contrastare con i valori liberali propri delle società ospitanti. In particolare, assume rilevanza la diversa connotazione della nozione stessa di medicina, intesa come prassi terapeutica, basata su principi, come il rispetto dell'autonomia del soggetto, sconosciuti in alcune comunità. Ciò comporta la necessità di valutare non solo l'adeguatezza del modello sanitario tipico delle società liberali, ma anche la forza delle prospettive etiche sulle quali esso si basa, prestando attenzione al diritto di perseguire i propri piani di vita senza impedimenti o costrizioni, con l'unico vincolo di non arrecare danno agli altri, quale condizione essenziale per la convivenza ${ }^{1}$. Assumendo il pluralismo dei valori e la varietà di posizioni morali sostanziali, nonché

\footnotetext{
* Dottoranda in Filosofia del Diritto Dipartimento dei Sistemi Giuridici dell'Università degli Studi di Milano-Bicocca 1 Cfr. P. Borsellino, Bioetica tra "morali" e diritto, Raffaello Cortina Editore, Milano 2009.
} 
la difficoltà di dare al "bene" una connotazione oggettiva e universalmente condivisa, si deve, dunque, preferire un modello che dia la priorità alla valutazione individuale relativa a cosa costituisca il proprio "bene".

In questo contesto si inserisce il tema delle mutilazioni genitali femminili, che emerge con sempre maggior urgenza a seguito del considerevole aumento dell'attività migratoria e della conseguente diversificazione dei sistemi etici e culturali, in nome dei quali gli individui pretendono di avere diritto a compiere scelte e a richiedere specifici trattamenti sanitari. Le MGF diventano un problema quando la richiesta di effettuare tali pratiche viene avanzata da alcuni gruppi sociali radicati nel contesto occidentale, in quanto considerata come un'istanza volta a fare il "bene" di chi ne è destinatario. Ci si domanda, dunque, fino a che punto tali pretese siano da accogliere e siano giustificabili. «Si possono, in particolare, ritenere giustificate e accoglibili richieste di interventi fatte in nome di un preteso diritto all'incondizionato rispetto della cultura minoritaria del richiedente, da parte della società di accoglienza, e delle sue varie istituzioni, in questo caso da parte delle istituzioni sanitarie, anche e soprattutto nel caso in cui tali pratiche appaiano in aperto contrasto con i fondamentali principi della società liberale e con la tutela della dignità e dell'integrità personale? E ancora, distinguere tra pratiche ammissibili e non ammissibili alla luce dei nostri principi significa inevitabilmente negare alle minoranze la loro identità culturale, incorrendo in una sorta di "imperialismo etico" >?? Oppure sussiste il rischio di incorrere in una forma di "relativismo etico", qualora si lasci a ciascun gruppo la libertà di decidere della bontà o della malvagità delle pratiche in esso attuate? In questo contributo si tenterà di far emergere profili di analisi nel tentativo di dare una risposta ai quesiti sollevati.

\section{Le mutilazioni genitali femminili come fatto sociale e culturale}

La prima classificazione di mutilazioni genitali femminili è stata elaborata da una Commissione tecnica sulle MGF riunita dall'Organizzazione Mondiale della Sanità (OMS) a Ginevra nel 1995, ed è accompagnata dalla descrizione della pratica e dalla sua valutazione

2 Ibid. p. 177. 
negativa da parte dell'Organizzazione medesima ${ }^{3}$. La definizione e classificazione che, tuttavia, vengono usate attualmente per riferirsi al fenomeno derivano dalla Dichiarazione Congiunta sulle MGF elaborata nel 1997 da OMS, UNICEF ed UNFPA, nella quale si afferma che: «Le mutilazioni genitali femminili comprendono tutte quelle procedure che comportano la rimozione, totale o parziale, dei genitali femminili esterni od altre alterazioni di questi organi, per motivi culturali od altre ragioni non terapeutiche. Queste procedure sono irreversibili, dannose per la salute delle donne che le subiscono ed hanno conseguenze che durano per tutta la vita ${ }^{4}$.

Prima ancora della definizione fornita dall'OMS, questo termine fu adottato nella terza conferenza del Comitato interafricano sulle pratiche tradizionali che colpiscono la salute di donne e bambini, tenutasi ad Addis Abeba nel 19905. L'impiego del termine "mutilazione" rafforza l'idea che questa sia una violazione dei diritti umani delle bambine e delle donne, e quindi aiuta a promuovere l'impegno nazionale e internazionale per il suo abbandono.

Per le comunità interessate, tuttavia, l'uso di questo termine può essere problematico a causa della sua forte connotazione negativa che viene percepita come uno stigma, un segno di disabilità riferito a corpi imperfetti, mutilati. "Mutilazione" è un termine che suscita imbarazzo anche nelle società occidentali per il suo connotato etnocentrico che reinventa i corpi dei migranti come qualcosa di diverso e grottesco rispetto al modello di corpo occidentale, creando un confine simbolico tra "noi" e "loro". Per descrivere la pratica, invece, le lingue locali in genere usano l'equivalente del termine "escissione", che possiede una minore connotazione valutativa, in quanto, comprensibilmente, i genitori rifiutano l'idea di sottoporre a mutilazione le proprie figlie.

La disparità di significati non è solo un problema etico, ma pri-

\footnotetext{
3 «La mutilazione genitale femminile è una pratica tradizionale profondamente radicata. Tuttavia si tratta di una forma di violenza con conseguenze negative sulla salute fisica e psicologica. E' inoltre una discriminazione nei confronti delle donne. L'OMS s'impegna per l'eliminazione di tutte le forme di MGF, con l'intento di attuare l'effettiva protezione e promozione dei diritti umani delle donne, compreso il diritto all'integrità fisica ed al raggiungimento di livelli ottimali di benessere fisico, mentale e sociale».WHO, Female Genital Mutilation. Report of a WHO Technical Working Group, Geneva, 17-19 July 1995.

4 WHO, Female Genital Mutilation: a joint WHO/UNICEF/UNFPA statement, Geneva, World Health Organization, 1997, p. 3.

5 Nel 1990 l'Inter-African Committee on Traditional Practices Affecting the Health of Women and Children (IAC) adottò il termine "mutilazioni genital femminili" per descrivere la procedura precedentemente indicata come "circoncisione femminile".
} 
ma di tutto è un problema epistemologico, dal momento che, utilizzando termini differenti, sembra che si faccia riferimento a pratiche differenti. Nella cultura occidentale il termine "mutilazione" ha un significato peggiorativo, riferito a una disabilità, a un corpo trasfigurato, che ha perso la sua integrità e armonia naturale. Per altre culture, al contrario, tali pratiche rappresentano un'operazione volta a rinforzare proprio l'integrità del corpo attraverso la rimozione di quelle parti degli organi genitali femminili che lo rendono impuro. A tale riguardo, ha cominciato ad affermarsi alla fine degli anni Novanta l'espressione female genital cutting (FGC, letteralmente "taglio dei genitali femminili"), che fa riferimento solo all'atto del tagliare, senza giudizi valutativi, e ha l'obiettivo di sottolineare il rispetto per le culture tradizionali che comprendono tra le proprie forme espressive tale pratica. Molte attiviste, africane e non, hanno, però, ravvisato un rischio nella scomparsa del riferimento alla mutilazione, come se venisse meno parte della motivazione all'abbandono della pratica, che sta proprio nel fatto che essa priva le donne di una parte funzionale del proprio corpo ed è espressione «di una disparità di genere profondamente radicata che assegna loro una posizione di subalternità nella società, e produce gravi conseguenze fisiche e sociali $>{ }^{6}$.

Nonostante le MGF siano generalmente percepite come una violenza di genere, volta a rappresentare l'assoggettamento delle donne al potere maschile, in alcune culture sono considerate un rite de passage, ovvero un rituale che possa guidare, controllare e regolare cambiamenti di status ${ }^{7}$. È un rito che segna la differenza tra i diversi momenti in cui è composta la vita umana, trasformandoli in percorsi ordinati dotati di un significato, in cui viene esplicitata la necessità di istanze di identità e di riconoscimento. In particolare, in determinati contesti, le MGF possono essere considerate un rito di iniziazione, attraverso il quale le bambine diventano donne mediante la trasformazione dell'identità sessuale in uno status sociale, riscattando il destino biologico legato al sesso e trasformandolo in

\footnotetext{
6 UNICEF. Centro di ricerca Innocenti (IRC), Cambiare una convenzione sociale dannosa: la pratica della escissione/mutilazione genitale femminile, Firenze, UNICEF. IRC, 2005.

7 Secondo Gerry Mackie, spesso le MGF non corrispondonosempre ad un rito di iniziazione, dal momento che in alcuni gruppi vengono effettuate durante l'infanzia, mentre in altre dopo il primo parto. Al contrario, in paesi come Senegal e Nigeria, le MGF sono associate a cerimonie, che celebrano l'entrata delle donne nella società. Cfr. G., Mackie,Female Genital Cutting: The Beginning of the End, in B. ShellDuncan and Y. Hernlund(eds), Female Circumcision: Multidisciplinary Perspectives, Lynne Reinner Publishers, Boulder, CO 2000, pp. 245-282.
} 
una "essenza sociale": la donna. Tuttavia, tali rituali hanno anche la funzione di marcare il confine tra l'identità femminile e quella maschile. Infatti, secondo alcune tradizioni, «alla nascita un bambino ha un'anima maschile e una femminile. L'anima femminile del maschio è sita nel prepuzio, l'anima maschile della femmina risiede nel clitoride. Un bambino, quindi, può diventare totalmente maschio o totalmente femmina solo se viene "tagliato" $>^{8}$.

Le MGF, intese come fatto sociale, sono considerate la porta di accesso alla propria comunità di appartenenza e costituiscono il punto di non ritorno che divide chi è dentro da chi è fuori. Sono, altresì, ritenute una "ferita simbolica" attraverso la quale ciascun gruppo scrive il proprio nome sul corpo, imprimendo un marchio che trasforma questi corpi in barriere culturali. Le MGF rappresentano un "confine etnico" che segna l'identità sociale dall'interno; un confine che si traduce nell'endogamia, ovvero nell'abitudine, ma più spesso nell'obbligo, di scegliere il partner all'interno del gruppo. In questo modo, le MGF assumono il particolare ruolo di pratiche di conservazione e di legittimità dell'identità etnica nei processi migratori. Infatti, in assenza di identità culturali di riferimento stabili, la necessità di ritenere i corpi femminili come muti depositari di un'insostituibile appartenenza collettiva diviene ancor più vincolante. Tali ferite rappresentano la memoria della comunità che trasforma le donne in portatrici di un complesso sistema simbolico attraverso il quale ogni gruppo etnico può riconoscersi ${ }^{9}$.

\section{Politiche di riconoscimento tra universalismo e rela- tivismo culturale}

È difficile negare l'esistenza di un danno oggettivo prodotto dalle MGF, sia livello biologico, in quanto tali pratiche sono generalmente eseguite in pessime condizioni igieniche, causando infezioni e, a volte, anche la morte, sia livello psicologico, dal momento che rappresentano l'espressione più paradigmatica della subordinazione della donna al potere maschile. Tuttavia, questa lettura non raccoglie un consenso universale e coloro che la sostengono vengono accusati di imperialismo culturale dai gruppi che praticano le MGF,

\footnotetext{
8 S. Saharso, Multicultural feminism: finding our way between universalism and antiessentialism, IPW Working Paper No. 3/2008, Insitut für Politikwissenschaft, Universität Wien, p.14.

9 Cfr. C. Pasquinelli, Infibulazione. Il corpo violato, Meltemi Editore, Roma 2007.
} 
i quali ritengono che nessuna cultura abbia il diritto di ritenersi superiore a un'altra, pretendendo che i propri valori vengano considerati universali. Tale punto di vista è sostenuto da alcune versioni di relativismo etico, secondo le quali non esistono valori universali e nessuna cultura ha l'autorità morale per giudicare l'eticità di pratiche e comportamenti appartenenti ad altre culture. In contrasto con il relativismo etico, l'universalismo ritiene che ciò che è giusto e ciò che è sbagliato, il bene e il male, siano universalmente connota$\mathrm{ti}$, indipendentemente dalle differenze culturali ${ }^{10}$.

Il problema che si vuole affrontare in questa sede, dunque, spinge a porsi l'interrogativo se la pratica delle MGF possa essere universalmente condannata oppure se possa essere giustificata in base a valori particolari non universalmente condivisi. Assumendo un punto di vista universalistico, sarebbe possibile condannare la pratica delle MGF in quanto contraria alla dignità umana e ai diritti fondamentali riconosciuti dalle dichiarazioni internazionali. Il contrasto tra relativismo e universalismo mette in luce il conflitto tra il rispetto delle tradizioni e dell'identità culturale, e dei diritti individuali, in particolare dei diritti di libertà e di autonomia ${ }^{11}$.

Per poter affrontare la questione dell'accoglibilità della richiesta di MGF, è necessario premettere alcune considerazioni sulla diversità culturale. La necessità di riconoscimento è alla base della maggior parte delle rivendicazioni poste in essere dalle minoranze, che avanzano non solo richieste di inclusione, ma anche la pretesa di esprimere l'identità culturale che le contraddistingue, mantenendo la propria diversità rispetto alla cultura di maggioranza. Il principale problema delle società liberali consiste nel definire le modalità di integrazione tra le diverse culture, assicurando al tempo stesso i diritti inviolabili di libertà sui quali esse sono fondate.

Ben difficilmente potrebbe dirsi liberale una società in cui non vi fosse spazio per il riconoscimento del diritto dell'individuo alla propria diversità culturale. In tal caso, come sostiene Taylor, si dovrebbe parlare di uno Stato "cieco alle differenze", altresì chiamato neutrale, che ignorando le differenze, assimila ciò che è diverso alla prospettiva etica dominante ${ }^{12}$. In questo modo, lo Stato non appare

\footnotetext{
10 Cfr. R. Macklin, Against relativism. Cultural diversity and the search for ethical universals in medicine, Oxford University Press, New York 1999.

11 Cfr. R. Sala, D. Manara, Nurses and Request for Female Genital Mutilation: cultural rights versus human rights, in Nursing Ethics, 2001, 8 (3), pp. 247-258.

12 Cfr. C. Taylor, The politics of recognition (1992), tr. it. La politica del riconoscimento, in C. Taylor, J. Habermas, Multiculturalismo. Lotte per il riconoscimento, Feltrinelli, Milano 1998, pp. 9-62.
} 
affatto neutrale, in quanto tende all'omologazione, costringendo i cittadini stranieri a rinunciare alla loro identità, a spogliarsi delle proprie differenze culturali per rivestire i panni della normalità della maggioranza ${ }^{13}$. L'inclusione delle minoranze nella società deve avvenire, invece, attraverso il riconoscimento pubblico delle differenze, in quanto espressione delle diverse identità.

Tuttavia, proprio nel rispetto dei diritti di libertà, l'individuo ha il diritto, non il dovere, di uniformarsi alle tradizioni del gruppo di appartenenza. In quest'ottica è opportuno distinguere, dunque, i diritti culturali dal diritto dell'individuo di scegliere se uniformarsi o meno a una determinata cultura. Riconoscere l'esistenza di diritti culturali comporta il rischio di valutare positivamente una cultura anche quando essa è imposta ai suoi membri e di contraddire, in questo modo, i diritti fondamentali delle società liberali. Il riconoscimento così inteso, definito anche "riconoscimento in senso forte", implica l'accoglimento della richiesta di una cultura di essere riconosciuta in quanto avente in sé valore ${ }^{14} \mathrm{e}$ comporta l'adozione della teoria etica denominabile "relativismo etico", che fa dipendere la giustezza di una pratica dalla conformità alle tradizioni appartenenti alla cultura di riferimento.

In questa sede, al contrario, si vorrebbe sostenere un "riconoscimento in senso debole", che implica il diritto dell'individuo a ricevere un trattamento da eguale nel rispetto delle sue differenze. In questo modo lo Stato liberale non tutela le differenze in quanto in sé buone o valide, ma in quanto scelte dall'individuo stesso. Come sostiene Kymlicka, la condizione a cui è soggetto il riconoscimento di un diritto risiede nella sua compatibilità con i diritti di libertà e di autonomia ${ }^{15}$. Ciò significa che si devono considerare inammissibili non solo le pratiche che opprimono e sfruttano persone appartenenti a gruppi differenti, ma anche le pratiche non già scelte, bensì imposte, in nome della tradizione dal gruppo agli individui che ne fanno parte ${ }^{16}$. In questa prospettiva, dunque, la pratica delle MGF non è accettabile, in quanto viola la libertà degli individui.

In aperto contrasto con Kymlicka, Kukathas sostiene che tutti gli

\footnotetext{
13 Il principio dell'eguale considerazione e rispetto è centrale nella riflessione di R. Dworkin, Taking rights seriously (1977), tr. it. I diritti presi sul serio, il Mulino, Bologna 1982.

14 Cfr. R. Sala, Curare la diversità, in G. Bonacchi (a cura di), Dialoghi di Bioetica, Carocci, Roma 2003, pp. 155-166.

15 Cfr. W. Kymlicka, Multicultural Citizenship (1995), tr. it. La cittadinanza multiculturale, il Mulino, Bologna 1999.

16 Cfr. P. Borsellino, Bioetica tra "morali" e diritto, op. cit.
} 
individui devono essere liberi di scegliere il proprio gruppo di appartenenza e di vivere nel rispetto dei termini prestabiliti dal gruppo $^{17}$. Lo Stato dovrebbe rispettare queste comunità culturali, anche qualora mettano in atto politiche illiberali, i cui effetti siano rivolti esclusivamente ai membri del gruppo, in quanto sono formate da individui liberi, i cui diritti di libertà di scelta devono essere a loro volta rispettati. Secondo Kukathas, infatti, in tali comunità non esiste alcun meccanismo di oppressione, dal momento che ogni individuo è titolare del diritto di essere libero di uscire dal gruppo. Tale accezione dei diritti individuali «fornisce autorità alla comunità culturale [...] e, se i membri della comunità culturale vogliono continuare a vivere secondo le loro credenze, la comunità di accoglienza non ha alcun diritto di intervenire per prevenire che essi agiscano in conformità con i loro diritti» ${ }^{18}$. Tuttavia, se un individuo facesse appello alla comunità ospitante contro una pratica messa in atto dal gruppo di appartenenza, lo Stato dovrebbe proteggere tale individuo. In riferimento al caso delle MGF, questa opzione riguarda esclusivamente il caso di donne adulte, che siano in grado di abbandonare il gruppo e di rivolgersi alla più ampia comunità politica, sostenendo anche i costi economici e sociali che ciò comporta ${ }^{19}$.

La proposta di Kukathas è problematica, proprio in ragione del concetto di partecipazione volontaria alle pratiche culturali, centrale nelle riflessioni dell'autore. Infatti, se un determinato gruppo ha il potere di controllare l'educazione dei suoi membri, essi difficilmente considereranno sbagliate le pratiche da questo prescritte. $\mathrm{Ci}$ si domanda, quindi, come potrebbe essere provata l'appartenenza volontaria ad una minoranza culturale qualora essa fosse una comunità chiusa, dotata del potere di effettuare pratiche illiberali senza l'interferenza dello Stato. Anche qualora si dimostrasse l'effettiva volontà del singolo individuo di far parte di una comunità, sarebbe opportuno prestare attenzione agli eventuali processi di persuasione e di indottrinamento da questa messi in atto. Inoltre, coloro che volontariamente decidessero di appartenere ad un gruppo potrebbero essere indotti a farlo per diverse ragioni (ad esempio, affinità

17 Cfr. C. Kukathas, Are there any cultural rights?, in Political Theory, 1992, 20 (1), pp.105-139; ora in W. Kymlicka (ed.), The rights of minority cultures, Oxford University Press, Oxford 1995, pp. 228-256.

18 C. Kukathas, Are there any cultural rights?, op. cit. p. 238 (traduzione propria).

19 Infatti, una donna, abbandonando il gruppo, oltre a non essere in grado di mantenere se stessa economicamente, potrebbe essere costretta a rinunciare ai propri figli. Spesso è la paura del "mondo esterno" a impedire alle donne di uscire dalla comunità di appartenenza. 
culturale, legami familiari, ecc.), senza, tuttavia, essere d'accordo sulla giustezza di determinate pratiche. Questi individui non sarebbero adeguatamente protetti dallo Stato, in quanto nella prospettiva di Kukathas si assume che, nel momento in cui si sceglie di far parte di un gruppo, si accetta qualsiasi pratica in virtù dell'appartenenza volontaria. Tale modello, fondato sul riconoscimento e sulla tolleranza di tutte le differenze, conduce, dunque, ad una sorta di "paradosso della vulnerabilità multiculturale", che ha luogo quando il gruppo difende certe pratiche che mettono sistematicamente a rischio i diritti fondamentali degli individui che vi appartengono ${ }^{20}$.

Alla luce di queste considerazioni, il modello che appare più difendibile non è quello indifferente nei confronti delle diversità e neppure quello che giustifica la tolleranza delle differenze in nome del principio del rispetto delle culture. Il modello difendibile è, invece, quello in grado di spiegare «in che modo i diritti delle minoranze coesistono con i diritti umani e in che modo i diritti delle minoranze vengono limitati dai principi di libertà individuale, democrazia e giustizia sociale ${ }^{21}$. Tale modello permette di realizzare il riconoscimento delle culture minoritarie tenendo conto del diritto all'autonomia e della dignità dell'individuo; «la valorizzazione delle differenze culturali avviene, cioè, al solo fine di rispettare la libertà dei singoli di identificarsi con la cultura cui appartengono. In quest'ottica qualsiasi rivendicazione fatta in nome del gruppo, ma a discapito di coloro che vi appartengono, non è giustificata» ${ }^{22}$.

\section{Il caso italiano e la proposta del rituale simbolico}

È opportuno, dunque, interrogarsi su come dovrebbero comportarsi le società liberali a fronte della richiesta di MGF; se vietarle in nome dei diritti fondamentali di libertà e di autonomia oppure permetterle in nome del preteso diritto delle culture a conservare le proprie tradizioni. Le MGF rientrano certamente nella tradizione culturale di alcuni gruppi, tuttavia, un problema nasce dal momento che queste sono generalmente eseguite su individui non in grado di esprimere autonomamente il proprio consenso. È difficile immaginare, infatti, che le bambine scelgano liberamente di farsi mutilare.

20 Cfr. A. Schachar, On citizenship and multicultural vulnerability, in Political Theory, 2000, 28 (1), pp. 64-89.

21 W. Kymlicka, Multicultural Citizenship, op. cit., p.15.

22 R. Sala, Curare la diversità, op. cit., p. 163. 
La strada imboccata dall'Italia è stata quella della decisa e intransigente opposizione, sul piano giuridico, a ogni tentativo di apertura in direzione di tali pratiche. Infatti il Parlamento italiano, con la legge n.7 del 9 gennaio 2006, ha provveduto a tutelare la donna dalle pratiche di MGF, con lo scopo di prevenire, contrastare e reprimere pratiche intollerabili che colpiscono bambine e adolescenti, e che violano i fondamentali diritti della persona, primo fra tutti quello alla integrità fisica. Al codice penale è stato aggiunto l'art. 583-bis, che punisce con la reclusione da quattro a dodici anni chi, senza esigenze terapeutiche, cagiona una mutilazione degli organi genitali femminili. Le disposizioni di questo articolo si applicano, altresì, quando il fatto è commesso all'estero da cittadino italiano o da straniero residente in Italia.

Inoltre, la legge n.172 del 1 ottobre 2012, con la ratificazione della Convenzione di Lanzarote, prevede l'inserimento di un ulteriore comma all'art. 583-bis, secondo cui la condanna prevista per questo reato, nel caso in cui sia commesso dal genitore o dal tutore della bambina vittima, comporta la decadenza dall'esercizio della potestà genitoriale. L'articolo 583-ter del codice penale, infine, prevede che l'operatore sanitario, resosi colpevole del fatto, sottostà alla pena accessoria dell'interdizione dall'esercizio della professione da tre a dieci anni.

Tuttavia, tale strategia repressiva presta il fianco a numerosi rilievi critici, formulati sulla base di considerazioni pragmatiche e di efficacia, più che sulla base di considerazioni di principio. Infatti, il metodo adottato risulta inefficace per combattere le MGF, in quanto ha comportato il paradossale aumento delle mutilazioni clandestine, nonché la riluttanza delle donne a ricorrere al Servizio Sanitario Nazionale e la difficoltà a denunciare le violazioni delle norme menzionate. In queste circostanze, dunque, le usanze appaiono essere più vincolanti di ogni legge. La ragione risiede nel fatto che le sanzioni derivanti dalla trasgressione di tale usanza sono considerate più gravose per il singolo rispetto a quelle che conseguono dalla violazione della norma giuridica. Infatti, una sanzione che corrisponde all'esclusione dalla comunità è motivo di vergogna per l'intera famiglia, dal momento che nessun uomo sarà mai disposto a sposare una donna "impura"23.

Ci si domanda, dunque, se esista una via intermedia che cerchi di conciliare, almeno provvisoriamente, il principio del rispetto dei diritti individuali con quello del rispetto della cultura di apparte-

23 Cfr. C. Pasquinelli, Infibulazione. Il corpo violato, op. cit. 
nenza. Una soluzione al problema è stata proposta nel 2003 da un ginecologo italiano di origini somale, Omar Abdulcadir, che ha offerto alle donne somale residenti sul territorio italiano la possibilità di non mutilare le proprie figlie, sostituendo la mutilazione vera e propria con un rituale simbolico. Tale rituale consisterebbe in una piccola puntura sul clitoride, previa applicazione di una crema anestetizzante, volta a provocare la fuoriuscita di una goccia di sangue, a cui seguirebbe la consegna di un certificato comprovante l'avvenuta circoncisione ${ }^{24}$. In questo modo il rituale sarebbe salvo, senza, tuttavia, provocare dolore e danni permanenti.

Tale proposta è stata presentata al Comitato Etico dell'Ospedale di Careggi a Firenze ${ }^{25}$ e alla Commissione Regionale di Bioetica ${ }^{26}$, che, dopo una lunga discussione, hanno dato la propria approvazione. Tuttavia, prima che di poter essere adottata, tale proposta è stata fortemente criticata dall'opinione pubblica in ragione del fatto che non sussistono differenze tra il simbolo e la mutilazione vera e propria, dal momento che l'inviolabilità del corpo è un valore universale che non può essere messo in discussione in nome della riduzione del danno ${ }^{27}$. Nell'appello all'integrità del corpo si può rinvenire il ribrezzo unanime nei confronti di rituali considerati barbarici, in quanto permettono agli uomini di mantenere il controllo simbolico sulla sessualità femminile. Ciò che conta sembra essere esclusivamente l'implicita conferma che possiamo ottenere dalla nostra rassicurante integrità del corpo, a cui affidiamo la salvaguardia dei nostri valori civili in opposizione alla loro arretratezza tribale.

Ma dove possiamo trovare la certezza relativa all'integrità del corpo? Alla base di questa credenza è possibile individuare una con-

\footnotetext{
24 Ibid.

25 Si veda il verbale n.12/03 della seduta del Comitato Etico Locale dell'Azienda ASL 10 di Firenze del 5 dicembre 2003.

26 Si veda il "Parere della Commissione Regionale di Bioetica" espresso nella seduta del 9 marzo 2004 relativamente alla "Prevenzione delle Mutilazioni Genitali Femminili: liceità etica, deontologica e giuridica della partecipazione dei medici alla pratica di un rito alternativo".

${ }_{27}$ A Seattle nel 1996 si presentò un caso simile a quello italiano, quando l'Harborview Medical Center manifestò sensibilità nei confronti della situazione culturale delle donne somale immigrate. Al fine di prevenire un danno permanente e di evitare il rischio di MGF clandestine, il Centro Medico propose alle donne somale una soluzione di compromesso, simile a quella avanzata dal Dott. Abdulcadir in Italia. Quando la proposta divenne pubblica, venne considerata un oltraggio intollerabile, volto a mantenere le donne in una posizione di sottomissione rispetto agli uomini, e la proposta non poté trovare attuazione. Cfr. D.S. Davis, Male and Female Genital Alteration: a Collision Course with the Law?, in Health Matrix: Journal of Law-Medicine, 2001 (11).
} 
cezione naturalistica del corpo che nasconde le manipolazioni culturali attraverso cui ogni cultura costruisce diverse idee di e sul corpo. Se su ogni corpo è impresso il marchio indelebile della cultura di appartenenza, allora dovremmo ritenere che non si possa propriamente parlare di naturalità dei corpi. L'integrità non è altro che una costruzione culturale particolare dei nostri corpi, spesso inconsapevole della loro dimensione simbolica, che è molto facile da decifrare in altri corpi, in quanto oggettivata dal nostro punto di vista naturalistico. Sia i nostri corpi, sia i corpi degli altri sono simboli che rispecchiano l'asimmetria delle relazioni di genere peculiari alle società di appartenenza. «Simboli che si iscrivono sui corpi predisponendoli a destini di autonomia o di oppressione. Più spesso corpi assoggettati che liberi. Sia che assumano la forma di una atavica soggezione alla più oscura delle tradizioni sia che si presentino alle performance umilianti del machismo mediatico. [...] Per ogni cultura sono infatti le convenzioni, i valori e i simboli a decidere quale è la soglia oltre la quale un comportamento o un intervento sul corpo diventa una violazione della sua integrità ${ }^{28}$.

L'efficacia simbolica della circoncisione alternativa potrebbe essere una soluzione pragmatica, seppur temporanea, volta a tutelare i diritti delle donne e a prevenire mutilazioni clandestine. Allo stesso tempo, dovrebbero essere implementati programmi sociali ed educativi in grado di supportare le donne nel loro processo di integrazione, senza imporre loro delle scelte obbligate, ma fornendo gli strumenti volti a facilitare l'adozione di decisioni autonome. Lo scopo non deve essere quello di proibire una pratica che viene considerata sbagliata in nome dei valori liberali, bensì quello di rendere autonome le donne e di aiutare le loro figlie a diventare donne in una società multiculturale, libere da qualsivoglia imposizione.

$\overline{28}$ C. Pasquinelli, Infibulazione. Il corpo violato, op. cit., pp. 15-16. 


\section{Bibliografia}

Borsellino P., Bioetica tra "morali" e diritto, Raffaello Cortina Editore, Milano 2009.

Davis D.S., Male and Female Genital Alteration: a Collision Course with the Law?, in Health Matrix: Journal of Law-Medicine, 2001 (11).

Dworkin R., Taking rights seriously (1977), tr. it. I diritti presi sul serio, il Mulino, Bologna 1982.

Kukathas C., Are there any cultural rights?, in Political Theory, 1992, 20 (1), pp.105-139.

Kymlicka W., Multicultural Citizenship (1995), tr. it. La cittadinanza multiculturale, il Mulino, Bologna 1999.

Mackie G., Female Genital Cutting: The Beginning of the End, in ShellDuncan B. and Hernlund Y. (eds), Female Circumcision: Multidisciplinary Perspectives, Lynne Reinner Publishers, Boulder, CO 2000.

Macklin R., Against relativism. Cultural diversity and the search for ethical universals in medicine, Oxford University Press, New York 1999.

Pasquinelli C., Infibulazione. Meltemi Editore, Roma 2007.

Saharso S., Multicultural feminism: finding our way between universalism and anti-essentialism, IPW Working Paper No. 3/2008, Insitut für Politikwissenschaft, Universität Wien.

Sala R., Curare la diversità, in Bonacchi G. (a cura di), Dialoghi di Bioetica, Carocci, Roma 2003.

Sala R., Manara D., Nurses and Request for Female Genital Mutilation: cultural rights versus human rights, in Nursing Ethics, 2001, 8 (3), pp. 247258.

Schachar A., On citizenship and multicultural vulnerability, in Political Theory, 2000, 28 (1), pp. 64-89.

Taylor C., The politics of recognition (1992), tr. it. La politica del riconoscimento, in Taylor C., Habermas J., Multiculturalismo. Lotte per il riconoscimento, Feltrinelli, Milano 1998.

UNICEF. Centro di ricerca Innocenti (IRC), Cambiare una convenzione sociale dannosa: la pratica della escissione/mutilazione genitale femminile, Firenze, UNICEF. IRC, 2005.

WHO, Female Genital Mutilation. Report of a WHO Technical Working Group, Geneva, 17-19 July 1995.

WHO, Female Genital Mutilation: a joint WHO/UNICEF/UNFPA statement, Geneva, World Health Organization, 1997. 


\section{CONTRATTI FLESSIBILI E IL BENESSERE FAMIGLIARE. CASO DI STUDIO: LE SCUOLE DELL'INFANZIA DEL COMUNE DI MILANO}

RicCARDO Bonato*

Chiara Caulo**

\section{Premessa}

La famiglia è il cuore del mondo affettivo, qui si sviluppano relazioni di reciprocità e di dono che aiutano a proteggere l'individuo, rassicurandolo rispetto alle necessità materiali, in una prospettiva di futuro.

Ogni componente della famiglia è responsabile del benessere degli altri ed è influenzato nel suo benessere, o malessere, dall'appartenere a un gruppo che opera in modo funzionale o disfunzionale.

La famiglia, vista come "seminarium civitatis", è l'istituzione che crea le fondamenta per l'evoluzione economica e valoriale della società: se le famiglie sono funzionali nella trasmissione e nella creazione di capitale materiale, spirituale e culturale, le future generazioni saranno ricche di beni materiali, spirituali e culturali.

Un elemento che influenza la famiglia è la dimensione lavorativa dei genitori, in quanto l'individuo armonizza le sue risorse economiche (retributive, di tutela e di welfare) e di tempo (libero, di lavoro

\footnotetext{
* Dottorando in Sociologia del Diritto Dipartimento dei Sistemi Giuridici dell'Università degli Studi di Milano-Bicocca ** Laureata in Psicologia

Dipartimento di Psicologia dell'Università degli Studi di Milano-Bicocca
} 
e di cura) con gli altri membri del nucleo.

Le regolazione dei rapporti di lavoro nel nostro Paese è stata oggetto di importanti riforme atte a dare soddisfazione ad una necessità del tessuto economico: il bisogno di strumenti contrattuali atti a rendere flessibile l'organizzazione del personale al fine di orientare, con minori costi, la produzione di beni e servizi rispetto alle esigenze mutevoli della domanda di mercato.

Si rende, quindi, necessaria una riflessione rispetto alle conseguenze sociali della "flessibilizzazione" del mercato del lavoro: i mutamenti economici e normativi si riversano inevitabilmente nelle vite reali delle persone, cambiandone i tratti.

Il differente trattamento dei lavoratori atipici include anche gli istituti a tutela della maternità.

Questo studio avanza l'ipotesi che il contratto di assunzione dei genitori possa influenzare lo sviluppo linguistico del bambino che nasce all'interno della famiglia, in quanto tale relazione viene mediata dalla possibilità effettiva di utilizzo da parte dei genitori degli istituti posti a tutela della maternità (quali i permessi di allattamento), in modo formalmente eguale per ogni contratto di lavoro subordinato, ovvero in modo già normativamente diverso per alcuni contratti di lavoro parasubordinato.

La normativa dispone, infatti, che le lavoratrici a tempo indeterminato, le lavoratrici apprendiste, le lavoratrici a tempo determinato (Art. 6 del D.Lgs. 368/2001) e le lavoratrici iscritte alla gestione separata (Art. 64 del .Lgs. 151/2001) abbiano i medesimi diritti di tutela della maternità, ma l'efficacia di tali norme è condizionata dalla necessità per la lavoratrice atipica di mantenersi produttiva per il datore di lavoro con lo scopo di essere riconfermata alla scadenza del termine del contratto.

\section{Le principali tutele alla lavoratrice-madre}

Le tutele della maternità in Italia sono differenti tra le lavoratrici a seconda del tipo di rapporto di lavoro in corso e hanno diverse sfumature in base al contratto collettivo di settore applicato (CCNL).

Il Testo Unico sulla maternità e paternità, ex art. 1 D.Lgs. 151/2001 (in seguito TU) racchiude la disciplina normativa riguardo «i congedi, i riposi, i permessi e la tutela della lavoratrici e dei lavoratori connessi alla maternità e alla paternità di figli naturali, 
adottivi e in affidamento, nonché il sostegno economico alla maternità e alla paternità».

Le principali tutele racchiuse dal Testo Unico ${ }^{3}$ sono

- divieto di discriminazione tra lavoratori in base allo stato matrimoniale o di famiglia;

- divieto di licenziamento della lavoratrice madre o del lavoratore padre;

- sospensioni retribuite o non retribuite dall'attività lavorativa per esigenze di cura del bambino.

Rispetto al tema trattato, assumono particolare rilevanza il divieto di licenziamento e le sospensioni dell'attività lavorativa.

Secondo l'art. 54 del TU, è fatto divieto di licenziamento della lavoratrice madre per il periodo intercorrente tra il concepimento del figlio e il compimento di un anno di età del bambino. A questo fine non è rilevante che il datore di lavoro sia stato informato dello stato di gravidanza della lavoratrice.

Il divieto di estromissione dal posto di lavoro non opera in caso di scadenza del termine del contratto, di cessazione dell'attività aziendale o di un suo ramo autonomo, e neppure in caso di licenziamento per colpa grave della lavoratrice.

Appare evidente che il presupposto - logico prima che operativo - che deve necessariamente sussistere affinché la previsione del divieto di licenziamento possa produrre effettivamente il suo scopo (quello di scongiurare che la maternità, provocando una temporanea improduttività della lavoratrice, sia la reale causa che induca il datore di lavoro a far cessare il rapporto di lavoro) è la prevista protrazione nel tempo del rapporto di lavoro. Tale caratteristica, come noto, è propria solo del rapporto di lavoro subordinato a tempo indeterminato, con la conseguenza che in tutti i rapporti di lavoro c.d.. flessibili (eg. contratto a termine, contratto di apprendistato, contratto a progetto), essendo caratterizzati da un orizzonte temporale limitato e predeterminato, l'evento maternità induce (nella quasi totalità dei casi) il datore di lavoro a provocare di fatto (senza necessità di licenziamento, ma semplicemente astenendosi dal rinnovare il rapporto) la cessazione del rapporto di lavoro.

Pertanto, si può sinteticamente dire che la durata temporale li-

3 Le tutele alla maternità descritte fanno riferimento alla formulazione normativa del Testo Unico in vigore nel periodo novembre 2012-marzo 2013, durante il quale sono stati raccolti i dati dello studio quantitativo che completerà l'analisi. 
mitata del rapporto lavorativo che caratterizza le tipologie di lavoro c.d. flessibili impedisce al divieto di licenziamento per ragioni di maternità di assolvere la finalità protettiva che gli è propria.

In aiuto alle specifiche necessità di cura del nascituro o del neonato, la legge dispone la possibilità o l'obbligo di periodi di sospensione dall'attività lavorativa.

Riguardo alle astensioni dall'attività lavorativa, la regolazione di questi istituti è differente rispetto al contratto di lavoro, ovvero, pur avendo in alcuni casi la medesima regolazione, c'è una possibilità di effettivo utilizzo differente.

Riguardo al contratto di lavoro subordinato a tempo indeterminato, la lavoratrice madre ha l'obbligo di astenersi dall'attività lavorativa per i due mesi che precedono il parto e per i successivi tre (art. 16 TU). E' data facoltà alla lavoratrice, nel rispetto del suo stato di salute, di ritardare l'inizio del congedo per maternità ad un mese prima del parto presunto e, corrispettivamente, di prolungarlo per i quattro mesi successivi alla nascita (art. 20 TU).

Il congedo di maternità dà luogo ad un'indennità pari all'80\% della retribuzione base.

Un secondo periodo di astensione dal lavoro può essere goduto facoltativamente da ciascun genitore nei primi otto anni di vita del figlio, per un massimo di 6 mesi, fruibili anche in modo frazionato (art. 32 TU). Tali congedi parentali non possono eccedere complessivamente, per entrambi i genitori, un massimo di 10 mesi. Per questi periodi è prevista un'indennità pari al 30\% della retribuzione base.

Inoltre, la lavoratrice madre ha diritto, prima del parto, a riposi per effettuare controlli prenatali (art. 14 TU) e, fino al primo anno di vita del bambino, a due riposi giornalieri per l'allattamento della durata di un'ora, cumulabili in un unico riposo della durata di due ore. Tali congedi sono pienamente retribuiti (art. 39 TU).

Le sospensioni dell'attività lavorativa che maggiormente impattano per continuità e durata della fruizione sono il congedo obbligatorio, il congedo facoltativo e i permessi giornalieri di allattamento.

I tre istituti in oggetto, pur consentendo tutti un periodo di sospensione dall'attività lavorativa alla madre, si differenziano per

- obbligatorietà della fruizione (il congedo obbligatorio è imposto dalla legge; il congedo facoltativo e i permessi giornalieri sono a discrezione del lavoratore);

- durata e continuità della fruizione (il congedo obbligatorio ha durata continuativa di 5 mesi a cavallo del parto; il congedo facoltativo è di durata di 6 mesi fruibili anche in modo 
frazionato durante i primo 8 anni di vita del figlio; i permessi giornalieri di allattamento riducono l'orario di lavoro di 2 ore al giorno costantemente durante tutto il primo anno di vita del bambino);

- retribuzione del periodo di sospensione (il congedo obbligatorio è remunerato all' $80 \%$ della normale retribuzione; il congedo facoltativo è remunerato al 30\% della normale retribuzione; i permessi giornalieri di allattamento sono remunerati al 100\% della normale retribuzione).

Specifica attenzione verrà data alla sospensione per allattamento, in quanto è un periodo continuativo, retribuito, ma facoltativo.

\section{Le tutele della maternità per le lavoratrici "flessibili"}

L'art. 60 del TU stabilisce che le lavoratrici a tempo parziale «beneficiano dei medesimi diritti di un dipendente a tempo pieno comparabile, per quanto riguarda la durata dei congedi previsti dal presente testo unico. Il relativo trattamento è riproporzionato in ragione della ridotta entità della prestazione lavorativa».

Nella sostanza si ha una riduzione del trattamento economico dei periodi di congedo secondo la percentuale di lavoro part-time.

Con riferimento al contratto a termine, l'art. 6 del D.Lgs. $368 / 2001$ enuncia un generale principio di parità di trattamento dei lavoratori a tempo determinato con quelli a tempo indeterminato, assicurando ai primi, proporzionalmente al periodo lavorato, "le ferie, la gratifica natalizia, il trattamento di fine rapporto e ogni altro trattamento in atto nell'impresa" per i secondi, " inquadrati nello stesso livello in forza dei criteri di classificazione stabiliti dalla contrattazione collettiva" (c.d. lavoratori comparabili).

Ne deriva che, ad esempio, se all'interno del periodo triennale di durata del contratto a termine la lavoratrice rimarrà incinta, essa potrà godere degli stessi identici diritti della lavoratrice a tempo indeterminato; dovrà quindi fruire del congedo obbligatorio, astenendosi dal lavoro nei due mesi precedenti e nei tre mesi successivi al parto. Potrà quindi fruire dei permessi di allattamento quotidiani della durata di due ore ciascuno.

Tuttavia, è evidente che, essendo per il datore già la maternità in sé un evento che riduce drasticamente la produttività della lavora- 
trice - quando addirittura non ne comporta, nel periodo di congedo obbligatorio, un abbattimento in termini assoluti - la fruizione durante il primo anno di vita del bambino dei c.d. permessi di allattamento, pur costituendo un diritto della lavoratrice, sarà mal tollerata dal datore di lavoro, il quale è sì obbligato dalla legge a concedere tali permessi se la fruizione ne è richiesta, ma non è affatto obbligato a rinnovare il contratto di lavoro che di lì a poco scadrà naturalmente.

Pertanto, è verosimile affermare che il datore, potendosi facilmente "liberare" di una lavoratrice non produttiva (o non produttiva al massimo) come è la lavoratrice in maternità, eviterà alla scadenza di rinnovarle il contratto a termine, interrompendo in tal modo il rapporto di lavoro e privandola di qualsiasi tutela dal punto di vista economico.

E' allora fin troppo facile comprendere che la lavoratrice, avendo tutto l'interesse a conservare l'occupazione attraverso il rinnovo del contratto a termine, eviterà di chiedere la fruizione dei permessi di allattamento che pur gli spettano, addirittura, eviterà di rimanere incinta.

In questo si sostanzia il dramma che caratterizza la condizione precaria di tutti quei lavoratori/lavoratrici assunti con contratti di lavoro c.d. flessibili: la facile possibilità che ha il datore di lavoro di far cessare il rapporto di lavoro (ad esempio, attraverso il mancato rinnovo del contratto) finisce per incidere direttamente sulla concreta fruizione di tutte quelle tutele che la legge ha previsto (maternità, orario di lavoro straordinario, permessi, prescrizione crediti retributivi ecc. ecc.) in ragione della acclarata condizione di inferiorità socio-economica del lavoratore rispetto al datore di lavoro. Incide su di esse nella misura in cui il lavoratore, al fine di evitare l'interruzione del proprio rapporto di lavoro (evento questo che di per sé non sarebbe drammatico se le condizioni del mercato del lavoro non fossero così ingessate come lo sono attualmente) sarà indotto a non far valere tutti quei diritti che la legge ha previsto a sua protezione e tutela.

La conseguenza principale di questo impianto è che quei diritti il cui riconoscimento legislativo è avvenuto grazie a decenni di lotte collettive-sindacali volte a ottenere la progressiva emancipazione del lavoratore dalla sua condizione di debolezza endemica - finiscono per non essere più goduti dai lavoratori d'oggi, i quali, pertanto, essendo menomati di garanzie essenziali per lo sviluppo pieno della loro personalità ed individualità, assistono impotenti a un drastico e generalizzato regresso del livello di tutele rispetto a quello di cui hanno beneficiato i lavoratori appartenenti ad una generazione precedente. 
La normativa prevede un trattamento nella sostanza differenziato delle lavoratrici assunte con contratti di tipo parasubordinato o autonomo ma, è utile notare come il nocciolo di protezione, che tutte le categorie formalmente hanno, si concentri nel periodo di "astensione obbligatoria" da due mesi prima del parto a tre mesi dopo il parto.

Il trattamento economico non è parificato tra le diverse categorie, ma è possibile affermare che le quantità degli emolumenti sono paragonabili.

In secondo luogo, invece, risulta evidente la differenza di trattamento per altri istituti. Le lavoratrici a tempo indeterminato fulltime godono di maggiori tutele riguardo alla possibilità di sospensioni del rapporto di lavoro,

- nel primo anno di vita del figlio, di due ore al giorno (retribuite al 100\%);

- nei primi otto anni del figlio, di 6 mesi fruibili in modo anche frazionato (trattamento economico al 30\%);

- nei primi tre anni del figlio, dei giorni di malattia del bambino (non retribuito).

Tali tutele, pur essendo formalmente sancite dal testo normativo, all'atto pratico non sono presenti nei contratti flessibili, in quanto

- non esiste una norma che tutela la lavoratrice dall'estromissione ${ }^{4}$ alla scadenza del contratto; l'essere in servizio è condizione necessaria per godere delle sospensioni retribuite e non retribuite;

- la percentuale di retribuzione dei periodi in astensione per i contratti part-time è molto penalizzante per la lavoratrice flessibile.

Date queste premesse, è possibile ipotizzare un minor utilizzo delle sospensioni da parte delle lavoratrici con un contratto flessibile in servizio rispetto alle lavoratrici con un contratto a tempo indeterminato full-time, e, pertanto, una possibilità minore della madre di essere presente e prendersi cura del figlio nei primi anni della sua vita.

Specifica attenzione sarà data allo sviluppo del linguaggio del bambino, in quanto consolidati studi psicopedagogici comprovano l'importanza della quantità e qualità del tempo passato dalla madre con il figlio nell'apprendimento dell'uso della parola.

\footnotetext{
4 Perdita del posto di lavoro dovuta al decorrere di un termine di durata del contratto di lavoro.
} 
Il privilegiato rapporto tra madre (intesa come figura di riferimento) e figlio indica una possibile difficoltà di sostituire le sospensioni dell'attività lavorativa della madre con la fruizione di servizi all'infanzia o altri strumenti di welfare.

\section{Lo sviluppo del linguaggio del bambino}

Nel corso della vita un bambino sviluppa abilità che lo portano dal primo vagito a pronunciare, in meno di un anno, le prime parole nella sua lingua madre.

Le capacità di percepire e produrre suoni articolati evolvono parallelamente e vi sono reciproche influenze ${ }^{5}$

Lo sviluppo della produzione vocale nel primo anno di vita del bambino non può che basarsi sulla produzione spontanea di suoni.

La progressione del bambino nella produzione di suoni è dovuta ad uno sviluppo fisico del suo apparto vocale e ad acquisite capacità fonetiche.

Il tratto vocale di un neonato è simile a quello di un primate adulto non umano:

- la laringe è molto più in alto rispetto alla posizione che occupa nell'adulto;

- la lingua è molto grande rispetto alla cavità orale ed è quindi dotata di una motilità limitata;

- il canale orofageo è tale per cui l'epiglottide e la velofaringe sono molto vicine, dunque il neonato ha poca possibilità di espirazione se non con la bocca aperta.

Per poter produrre tutti i suoni necessari a comporre le parole è necessario controllare e coordinare i movimenti di laringe, glottide, palato molle, mandibole, labbra e lingua.

La pronuncia di una semplice frase implica la messa in moto e la coordinazione di più di cento muscoli, inoltre il ciclo respiratorio dev'essere combinato e sincronizzato con l'attività della corde vocali.

Lo sviluppo di tale apparato è comunque abbastanza veloce, a 3 mesi il palato si abbassa, la lingua si allunga e la sua motilità si rafforza. A 5 mesi il controllo del ritmo respiratorio e della fonazione è già abbastanza buono.

Le fasi dello sviluppo del linguaggio si suddividono in fase prelinguistica e fase linguistica.

5 D’Odorico L., Lo sviluppo linguistico Editori Laterza, Roma-Bari, 2010 
Stark e altri ${ }^{6}$ hanno indicato, quale sequenza di variazione dello sviluppo funzionale e anatomico dell'apparato fono articolatorio nella fase prelinguistica, la seguente progressione:

- stadio 1- Suoni di tipo riflesso (0-2 mesi). In questo periodo i bambini producono quasi esclusivamente suoni legati a condizioni fisiologiche di disagio (come il pianto) o richieste di nutrimento o suoni di tipo vegetativo (come colpi di tosse). I suoni di pianto sono prodotti con la bocca aperta e quindi prevalentemente regressivi e sonori, cioè simili alle vocali;

- stadio 2-Suoni di benessere (2-4 mesi). I suoni prodotti in questo periodo hanno caratteristiche fonetiche che dimostrano un controllo volontario dell'apparto fonatorio e sono prodotti in situazioni di benessere, spesso durante il contatto visivo o fisico con la madre. Inizialmente sono prodotti come segmenti isolati, successivamente si articolano su serie più lunghe, separate da pause brevi.

- stadio 3- Gioco vocale (4-7 mesi). In questa fase i bambini acquisiscono un maggior controllo sulla laringe e sui meccanismi articolatori e questo li porta a produrre una varietà di suoni, prevalentemente vocalici, con variazioni di intensità e intonazione tali da dare all'ascoltatore l'impressione che il bambino stia "giocando" con il proprio apparto fonatorio. In questo periodo possono essere prodotte le prime consonanti;

- stadio 4- Lallazione canonica e reduplicata (7-12 mesi). Questo periodo può essere considerato il livello di sviluppo più avanzato raggiunto dal bambino in periodo prelinguistico; secondo alcuni autori questa fase segna l'inizio della fase linguistica: i bambini sono in grado di produrre sillabe che hanno le stesse caratteristiche di quelle presenti nelle lingue naturali. E' all'interno delle sequenze sillabiche che sono percepite le regolarità fonotattiche ed è la sillaba che veicola la maggior parte delle informazioni prosodiche. Ora i bambini possono riprodurre quanto ascoltato nei mesi precedenti.

L'approccio socio-interattivo, prevalente in letteratura, indica che le interazioni tra madre e bambino sono di per sé strutture comunicative in cui avviene uno scambio di messaggi che permette la mutua regolazione dei comportamenti ed ivi va ricercata la struttu-

\footnotetext{
$6 \quad$ Stark R.E., Bornstein, L.E., Demorest M.E., Vocal communication in the first 18 months of life, journal of Speech and Hearing Research, 36, 1993, pp. 548-558
} 
ra comunicativa su cui si fonda l'acquisizione del linguaggio ${ }^{7}$.

Il bambino costruisce con la madre, ovvero con la figura di riferimento che si prende principalmente cura di lui in assenza della madre, un sistema comunicativo incrementale che non è riconducibile alla semplice somma dei comportamenti separati delle due persone: i comportamenti reciprocamente si adattano per giungere ad un maggiore stato di benessere; la struttura comunicativa, chiamata in letteratura "dialogo", aiuta la madre a comprendere il figlio maggiormente di chiunque altro e il bambino a comprendere gli stimoli comunicativi della madre maggiormente di quelli di altre persone.

Nella fase prelinguistica, il bambino inizia a scambiare segnali ulteriori rispetto a quelli comportamentali: il bambino ha l'intenzione di comunicare qualcosa che ha un contenuto diverso dal mezzo usato per comunicare (a differenza, ad esempio, di fermare l'attività di suzione, ove la comunicazione e il mezzo coincidono) attraverso un codice convenzionale dotato di regole che debbono essere utilizzate per la corretta ricezione del messaggio.

La creazione del codice di regole convenzionale si costruisce progressivamente con la reciproca interazione tra bambino/adulto. Il rapporto madre/bambino è in questo privilegiato.

Un breve congedo di maternità è correlato a interazioni meno positive nella coppia madre-bambino ${ }^{8}$.

Il passaggio dalla comunicazione prelinguistica alla comunicazione linguistica non avviene all'improvviso, ma è mediata dalla produzione di varie "forme foneticamente stabili", cioè il bambino produce suoni che possono avere una maggiore o minore somiglianza con il linguaggio adulto.

La distinzione tra queste "pseudo-parole" non è sempre facile. Non sempre ciò che il genitore indica come "prima parola" è quello che uno studioso dell'acquisizione del linguaggio riconoscerebbe come tale.

Questo, come in seguito esposto, sarà un limite dell'analisi quantitativa svolta.

Vihman e McCune ${ }^{9}$ hanno elaborato una serie di criteri per distinguere tra parole e "pseudo parole".

Una parola per essere considerata tale deve

1) avere caratteristiche formali di produzione (somiglianza fonetica) simili alla forma adulta;

\footnotetext{
D’Odorico L., Lo sviluppo linguistico Editori Laterza, Roma-Bari, 2010

8 Clark, R., Hyde, J.S., Essex, M.J., \& Klein, M.H. (1997). "Length of maternity leave and quality of mother-infant interactions". Child Development, 68, 364-383.

9 Vi M.M., McCune I (1994), “When is a word a word?", Journal of child language, $21,517-42$.
} 
2) essere dotata di significato, cioè essere utilizzata almeno una volta in un contesto in cui è la più appropriata, ovvero non deve essere utilizzata in un contesto dove è inappropriata;

3) essere presente, anche con variazioni fonetiche minime, più volte nelle produzioni del bambino.

L'età in cui il normale sviluppo del linguaggio porta il bambino pronunciare la prima parola è precedente ai 15 mesi. Qualora questo avvenga successivamente, il bambino ha subito un rallentamento nello sviluppo linguistico.

Tra i 12 e i 15 mesi, i bambini hanno un ritmo di acquisizione di nuove parole lento ( 7 parole al mese di media $)^{10}$.

Tra il 15 e i 27 mesi, si verifica "l'esplosione del vocabolario", cioè il bambino inizia ad acquisire parole ad una velocità maggiore (50 parole al mese di media). Questi dati sono tratti da uno studio effettuato tramite la somministrazione mensile del questionario Primo Vocabolario del Bambino ${ }^{11}$.

\section{Il ruolo della madre nello sviluppo del linguaggio}

Diverse ricerche ${ }^{12}$ hanno dimostrato che esiste una relazione positiva tra la formazione di un legame di attaccamento sicuro ${ }^{13}$ con la madre e lo sviluppo del linguaggio. E' possibile ipotizzare che i bambini sicuri mettano in atto una esplorazione più attiva e complessa dell'ambiente, con un vantaggio nello sviluppo cognitivo e linguistico e che l'esperienza di interazioni mutualmente soddisfacenti permettono una precoce scoperta della valenza comunicativa del pro-

\footnotetext{
10 D’Odorico L., Carubbi S., Salerni N., Calvo V., Vocabulary developement in Italian Children; a lopngitudinal evaluation of quantitative and qualitative aspects. "Hournal of Child Language, 2001, 28, 351-371.

11 Caselli M.C., Casadio P., Il primo vocabolario del bambino, Franco Angeli, Milano, 1995.

12 Van Ijzendoorn M.H., Dijkstra J., Bus A.G., Attachemnt intelligence and language: a metanaysis., Social Development, 1995, 4, 115-128.

D’Odorico L., Salerni L., Cassibba R., Jacob V., Stability and change of material speech to infants from 6 to 20 monthes of age: a longitudinal study of its influence on eary stages of language acquisition, First language, 1999, 57, pp. 313-346.

13 Secondo Bowlby [1989] il bambino piò sviluppare con la figura di riferimento (caregiver) un attaccamento "sicuro" o "insicuro": un attaccamento di tipo sicuro si ha se il bambino sente di avere dalla figura di riferimento protezione, senso di sicurezza e affetto; in un attaccamento di tipo insicuro invece il bambino riversa sulla figura di riferimento comportamenti e sentimenti come instabilità, prudenza, eccessiva dipendenza, paura dell'abbandono.
} 
prio comportamento.

In letteratura è noto che il tipo di input linguistico a cui il bambino viene esposto in fasi precoci di sviluppo esercita una notevole influenza sul futuro sviluppo del linguaggio. In particolare, alcune ricerche hanno dimostrato che esiste una relazione positiva tra la formazione di un legame di attaccamento sicuro e lo sviluppo del linguaggio ${ }^{14}$, anche se non sono stati pienamente chiariti i processi attraverso i quali questo vantaggio si realizzerebbe.

Attualmente la letteratura converge nell'attribuire alla "responsività" materna ai segnali del bambino la causa del legame tra stile di attaccamento (sicuro o insicuro) e lo sviluppo linguistico del bambino, mentre ci sono dati contrastanti su quali siano gli aspetti specifici dello sviluppo del linguaggio del bambino che ne risultano maggiormente influenzati.

D'altra parte va sottolineato che una particolare strategia interattiva madre-bambino può essere efficace per lo sviluppo del linguaggio a una particolare età dell'infante, ma può diventare inutile o addirittura dannosa in periodi successivi. Ad esempio, una madre iperapprensiva può favorire la prima fase dello sviluppo del linguaggio del bambino, in quanto l'infante è costantemente sottoposto a stimoli linguistici (nominazioni), ma sfavorire la successiva fase di sviluppo in quanto dà meno spazio al bambino per sperimentare individualmente la produzione di suoni.

In generale, è noto che la possibilità di istaurare formati di attenzione condivisa e lo stabilirsi di interazioni triadiche (madrebambino-oggetto) sia particolarmente rilevante per l'acquisizione del linguaggio. In tal senso, assume un ruolo cruciale la quantità di tempo che la madre può dedicare agli scambi interattivi basti sulla denominazione degli oggetti.

Ad avvalorare tale ipotesi, ritroviamo lo studio di Bornstein e Rubby ${ }^{15}$ che ha riscontrato un più diffuso ritardo linguistico, al netto di complicazioni perinatali, in una popolazione di gemelli. Una possibile ipotesi di spiegazione fa riferimento alle limitate risorse di tempo e attenzione che i genitori di gemelli possono dedicare ai propri figli.

14 Salerni N., Calvo V., D’Odorico L. Influenze di ordine affettivo, relazionale e cognitivo nello sviluppo della competenza linguistica “, Giornale Italiano di Psicologia, 2001, XXVIII(3), pp. 463-484.

15 Bornstein M.H., Ruddy M., Infant attention and maternal stimulation: prediction of cognitive and linguistic development in singletons and twins", in H.Bouma, D.G. Bouwhuis (a cura di ), Attention and performance: control of language process, Hillsdale, NJ, Lawrence Erlbaum Associates, 1984, pp. 433-445. 
Il ruolo delle funzioni comunicative, realizzate attraverso gli enunciati materni nel favorire o meno lo sviluppo linguistico, ha secondo Longobardi ${ }^{16}$

- "funzione tutoria" che fornisce sostegno attraverso ripetizioni, espansioni e riformulazioni di quanto il bambino ha espresso;

- "funzione didattica" che trasmette conoscenze e ne verifica l'apprendimento;

- "funzione di conversazione" che permette di mantenere aperta la comunicazione, ad esempio tramite auto-risposte;

- "funzione di controllo" che orienta l'attenzione del bambino o la modifica;

- "funzione asincronica" che si caratterizza per comportamenti intrusivi della madre, quali sovrapposizioni alle verbalizzazioni del bambino.

Nella conversazione con i bambini, le madri utilizzano un linguaggio sostanzialmente diverso dal linguaggio utilizzato tra adulti ("motherese", ovvero CDS), caratterizzato da maggiore concretezza, ridondanza e semplificazione sintattica.

Molti studi convergono sulla conclusione che la quantità di linguaggio rivolto esplicitamente al bambino precedentemente ai 12 mesi è importante per facilitare la fase dello sviluppo del linguaggio ${ }^{17}$. Questo effetto è probabilmente dovuto a maggiore esposizione a diversi vocaboli e all'utilizzo in differenti situazioni dello stesso vocabolo.

\section{Il caso di studio: le scuole dell'infanzia del Comune di Milano}

Attraverso un'analisi quantitativa è stato possibile raccogliere dati utili per lo studio dell'impatto dei contratti flessibili sul lavoratore, sulla sua famiglia e sulle generazioni future.

La popolazione oggetto dell'analisi quantitativa è rappresentata

16 Longobardi E. Funzionecomunicativa del comportamento materno e sviluppo comunicativo-linguistico del bambino nel second anno di vita, Giornale Italiano di Psicologia, 19, 1992, 425-448.

${ }_{17}$ D'Odorico L., Salerni L., Cassibba R., Jacob V., Stability and change of material speech to infants from 6 to 20 monthes of age: a longitudinal study of its influence on eary stages of language acquisition, First language, 57, 313-346. 
dalle famiglie con un bambino di età compresa tra 0 e 3 anni che frequenta, nell'anno scolastico 2012/13, un nido o una scuola dell'infanzia del Comune di Milano. La popolazione è di 9936 unità. E' stata predisposta un'analisi attraverso un questionario a risposta multipla autocompilato diviso in cinque parti in base all'argomento delle domande. All'interno del questionario la parte terza ha rilevato le abilità acquisite dal bambino durante il suo sviluppo, tra cui l'età della prima parola dell'infante, mentre la parte quarta e quinta hanno rilevato la situazione lavorativa dei genitori. Il questionario è stato verificato attraverso un pre-test con la somministrazione a 5 famiglie.

Tenute in considerazione le circostanze pratiche di distribuzione, i caratteri in analisi e il tasso di dispersione, è stato ritenuto sufficiente costruire un campione della popolazione di famiglie di 334 unità. Il campione è stato stratificato, proporzionalmente alla popolazione suddivisa per tipologia del nido frequentato (comunale, comunale accreditato, privato accreditato o privato convenzionato), per zona territoriale e per classe di età del bambino (lattanti, medio/grandi e primavera).

A seguito della distribuzione di 334 questionari, avvenuta tra novembre 2012 e marzo 2013, i questionari raccolti, a fine marzo 2013, sono stati 327, di cui uno solo non compilato. I dati raccolti sono stati digitalizzati ed elaborati con il programma statistico SPSS.

\section{I limiti dello studio}

Il primo limite di questo studio è quello di porre l'ipotesi che la madre sia la figura di attaccamento primaria per il bambino. Tale ipotesi è la più diffusa, ma questo non esula dal fatto che possa avvenire che la figura di attaccamento primaria sia il padre ovvero un altro soggetto.

In secondo luogo, essendo uno studio di tipo esplorativo, esula dai suoi obiettivi originari quello di identificare secondo gli standard medici un deficit specifico del linguaggio, pertanto è auspicabile approfondire i dati emersi dal seguente studio attraverso l'utilizzo di test standardizzati per valutare le specifiche competenze linguistiche.

Inoltre, per natura stessa dello sviluppo linguistico risulta necessario attuare valutazioni successive al periodo [0-3 anni] preso in 
considerazione dalla ricerca per verificare l'andamento nel tempo dello svantaggio linguistico rilevato nel campione. Infine, per aumentare l'attendibilità e la significatività dei dati in questione occorrerebbe ampliare l'aria geografica del campionamento.

Da ultimo, il risultato ottenuto in questo studio potrebbe essere condizionato da variabili esterne al modello analizzato, è necessario uno studio di approfondimento che depuri il risultato ottenuto da tutti i fattori di rallentamento nello sviluppo del linguaggio del bambino. La scelta di non includere i liberi professionisti nella ricerca è legata a due ordini di ragioni. In primis, la regolazione delle tutele della maternità poste dall'ordinamento è differente per i liberi professionisti, per i quali non esistono alcuni degli istituti analizzati (ad esempio i permessi di allattamento), ovvero le categorie caratterizzanti un rapporto di lavoro perdono senso (eg. il part-time). In secondo luogo, i contratti atipici (oggetto della ricerca) sono di tipo subordinato, ovvero parasubordinato e, quindi, sono estranei alla libera professione.

In conformità con la letteratura, il livello di significatività dei risultati statistici è posto al 95\% (sig.<0,050).

\section{I risultati}

Riguardo all'obiettivo di ricerca, questo studio avanza l'ipotesi che il contratto di assunzione dei genitori possa influenzare anche lo sviluppo linguistico del bambino che nasce all'interno della famiglia.Tale relazione viene mediata dalla possibilità effettiva di utilizzo da parte dei genitori degli istituti posti a tutela della maternità, in modo formalmente eguale per ogni contratto di lavoro subordinato, ovvero in modo già normativamente diverso per alcuni contratti di lavoro parasubordinato.

Il privilegiato rapporto tra madre e figlio indica una possibile difficoltà di sostituire le sospensioni dell'attività lavorativa della madre con la fruizione di servizi all'infanzia o altri strumenti di welfare.

I contratti di lavoro atipici e lo sviluppo linguistico

Il segmento di campione utilizzato per condurre questa analisi, composto da 203 famiglie, è rappresentato dai nuclei in cui la madre è lavoratrice non libera professionista. Delle 203 madri incluse 
nell'analisi 171 sono assunte a tempo indeterminato e 32 sono lavoratrici atipiche.

Rispetto allo sviluppo del linguaggio, l'età in cui il normale sviluppo del linguaggio porta il bambino pronunciare la prima parola è precedente ai 15 mesi. Qualora questo avvenga successivamente, il bambino ha subito un rallentamento nello sviluppo linguistico. Dei 203 bambini inclusi nello studio, 142 hanno detto la prima parola prima dei 15 mesi, mentre 61 hanno subito un rallentamento dello sviluppo.

Nonostante la maggiore distribuzione dei bambini con un ritardo nella categoria delle madri con contratto atipico, il test chi quadrato $\left(x^{2}=2,021 ; \mathfrak{p}=0,155\right)$ non raggiunge il valore minimo della significatività statistica (sig. > 0,050).

La significatività asintotica della tavola di contingenza con cui abbiamo analizzato la relazione è stata calcolata attraverso l'indice del Chi quadrato di Pearson.

\begin{tabular}{|l|l|l|l|l|}
\hline $\begin{array}{l}\text { Tipologia } \\
\text { contrattuale } \downarrow\end{array}$ & $\begin{array}{l}\text { Età della prima } \\
\text { parola } \rightarrow\end{array}$ & $\begin{array}{l}\text { Età } \\
\text { inferiore } \\
\text { a 15 mesi }\end{array}$ & $\begin{array}{l}\text { Età supe- } \\
\text { riore a 15 } \\
\text { mesi }\end{array}$ & Totale \\
\hline $\begin{array}{l}\text { Contratto a } \\
\text { tempo } \\
\text { indetermi- } \\
\text { nato }\end{array}$ & Conteggio & 123 & 48 & 171 \\
\hline & Percentuale & $71,9 \%$ & $28,1 \%$ & $100,0 \%$ \\
\hline $\begin{array}{l}\text { Contratto } \\
\text { atipico }\end{array}$ & Conteggio & 19 & 13 & 32 \\
\hline & Percentuale & $59,4 \%$ & $40,6 \%$ & $100,0 \%$ \\
\hline Totale & Conteggio & 142 & 61 & 203 \\
\hline & Percentuale & $70,0 \%$ & $30,0 \%$ & $100,0 \%$ \\
\hline
\end{tabular}

Tabella 8.8: Tavola di contingenza, tipologia contrattuale di assunzione/Età della prima parola del bambino (segmento di campione dei genitori-donna lavoratrici non libere professioniste con un bambino di età superiore a 15 mesi di 203 unità).

E' necessario approfondire questo dato ricercando delle variabili di mediazione: la diversa regolazione della tutela della maternità tra i contratti atipici e i contratti a tempo indeterminato (permessi giornalieri) potrebbe aver influito sullo sviluppo del linguaggio del bambino. 


\section{I permessi giornalieri e lo sviluppo linguistico}

Nella popolazione d'analisi si osserva che le lavoratrici atipiche hanno un accesso ostacolato alle tutele per la maternità: solo il 25,7\% delle lavoratrici atipiche utilizza i permessi giornalieri (allattamento), contro il 59,6\% delle lavoratrici a tempo indeterminato. L'utilizzo dei permessi di allattamento, inoltre, sembra influenzare lo sviluppo del linguaggio del bambino: i figli delle madri che non utilizzano i permessi rilevano nel $41,5 \%$ un rallentamento nello sviluppo del linguaggio, da confrontare con il 25,3\% dei figli delle madri che li utilizzano.

\begin{tabular}{|l|l|l|l|l|}
\hline $\begin{array}{l}\text { Utilizzo dei } \\
\text { permessi di } \\
\text { allattamento } \downarrow\end{array}$ & $\begin{array}{l}\text { Età della } \\
\text { prima } \\
\text { parola } \rightarrow\end{array}$ & $\begin{array}{l}\text { prima } \\
\text { dei 15 } \\
\text { mesi }\end{array}$ & $\begin{array}{l}\text { dopo } \\
\text { i 15 } \\
\text { mesi }\end{array}$ & totale \\
\hline $\begin{array}{l}\text { No, non posso/ } \\
\text { voglio goderne }\end{array}$ & Conteggio & 38 & 27 & 65 \\
\hline & Percentuale & $58,5 \%$ & $41,5 \%$ & $100,0 \%$ \\
\hline $\begin{array}{l}\text { Sì, ne ho } \\
\text { usufruito }\end{array}$ & Conteggio & 71 & 24 & 95 \\
\hline & Percentuale & $74,7 \%$ & $25,3 \%$ & $100,0 \%$ \\
\hline Totale & Conteggio & 109 & 51 & 160 \\
\hline & Percentuale & $68,1 \%$ & $31,9 \%$ & $100,0 \%$ \\
\hline
\end{tabular}

Tabella 1: tabella di contingenza: fruizione dei permessi di allattamento da parte del genitore-donna/età della prima parola del bambino (genitori-donna lavoratrici non libere professioniste che non usufruivano di altri periodi di sospensione dell'attività lavorativa e con un bambino di età superiore a 15 mesi di 160 unità).

La significatività della relazione espressa dalla tavola di contingenza (tab. 3) misurata con il Chi quadrato di Pearson, risulta superiore al livello di significatività ( $\mathrm{p}=0,030 ;$ sig. $<0,05 \%$ ). I dati raccolti sono sufficienti a supportare una significativa analisi quantitativa.

La regressione logistica dei dati raccolti ha portato alla conclusione che passando la lavoratrice-madre dal gruppo [ha goduto del permesso di allattamento] a [non ha goduto del permesso di allattamento], la possibilità che suo figlio appartenga al gruppo [Età della prima parola oltre i 15 mesi] è del $47,6 \%$ più alta. Tale risultato è significativo ( $p=3,1 \%$; sig. $<0,05 \%)$. 


\section{Conclusioni}

L'analisi sul campo sembra confermare l'ipotesi teorica: l'assunzione con un contratto atipico della madre potrebbe rallentare lo sviluppo del linguaggio del bambino da 0 a 3 anni e tale relazione viene mediata dalla difficoltà delle lavoratrici atipiche di utilizzare i permessi giornalieri di allattamento.

L'allattamento rappresenta per sua natura una situazione preferenziale per instaurare fenomeni di attenzione condivisa e interazioni triadiche, le quali rappresentano degli aspetti necessari per lo sviluppo socio-cognitivo del bambino, in particolare per ciò che concerne lo sviluppo linguistico.

Il tipo di contratto di lavoro della madre può esercitare almeno un parziale effetto sullo sviluppo linguistico del figlio, nella misura in cui il tipo di contratto di lavoro influenza la possibilità di usufruire dei permessi di lavoro giornalieri per l'allattamento. L'utilizzo dei permessi di lavoro giornalieri per l'allattamento può essere identificato come fattore protettivo per il successivo sviluppo linguistico del bambino. 


\section{Bibliografia}

Altieri G., "Dalle collaborazioni coordinate e continuative al lavoro a progetto: cosa cambia nel mercato del lavoro italiano" In M. Pallini (a cura di), Il lavoro a progettoin Italia e in Europa. pp. 19- 92, Il Mulino, Bologna, 2006

Barbagli M., Saraceno C., Lo Stato delle famiglie in Italia, pp. 7-26, Il Mulino, Bologna, 1997

Barbieri P., Reyneri E.," Il lavoro instabile a Milano e in Lombardia." In M. Belloni, M. e Carcano Marco (a cura di), Il lavoro atipico a Milano e provincia, Franco Angeli, Milano, 2007

Barbieri P., Scherer S. (2005). "Le conseguenze sociali della flessibilizzazione del mercato del lavoro in Italia. Differenze territoriali, fattori strutturali e rischi di esclusione sociale. "Stato e Mercato, 74

Barbieri P., Scherer S. (2007). "Vite svendute. Uno sguardo analitico sulla costruzione sociale delle prossime generazioni di esclusi." Polis, 21 (3), 431-459

Bauman Z., "Liquid Modernity”, Polity Press, Cambridge, 2000. (2002), "Informal labour market governance: The case of the British and German media production industries. "Work, Employment and Society, 16, 27-46

Bertolini S., Il lavoro atipico e le sue strategie, Libreria Stampatori, Torino, 2002

Bertolini S., Strumenti concettuali per l'analisi del mercato del lavoro atipico: riflessioni ed esperienze di ricerca, Sociologia del lavoro, 97, 2005, pp. 80-104

Bertolini S., Muehlberger U., The organizational governance of work relationships between employment and self-employment, Socio-Economic Review, 6 (3), 2008, pp. 449-472

Booth A.L. e altri, Temporary Jobs: Stepping Stones or Dead Ends?, Economic Journal, 112, 2002, pag. 480

Bornsteim M.H., Ruddy M., Infant attention and maternal stimulation: prediction of cognitive and linguistic development in singletons and twins, in H.Bouma, D.G. Bouwhuis (a cura di ), Attention and performance: control of language process, Hillsdale, NJ, Lawrence Erlbaum Associates, 1984, pp 433-445

Bowlby J., Attaccamento e perdita, Vol. I, II, III, ed. tradotta (1999), Bollati Boringhieri, Torino, 1989

Carcano (a cura di), Il lavoro atipico a Milano e provincia, ISMO, 
Milano, 2007

Caselli M.C., Casadio P. Il primo vocabolario del bambino, Franco Angeli, Milano, 1995

Cattaneo, A., A. Yngve, et AL. Protection, promotion and support of breastfeeding in Europe: current situation." Public Health Nutrition, 2005, 8(1): pp 39-46.

Chatterj P. and S. Markowitz Does the length of maternity leave affect maternal health?, Southern Economic Journal 72, 2005, pp 16-41.

Clark, R., Hyde, J.S., Essex, M.J., \& Klein, M.H., Length of maternity leave and quality of mother-infant interactions, Child Development, 68, 1997, pp. 364-383.

Commission of the European Communities, Assessment of the Implementation of the 2001 Employment Guidelines, Commission of the European Communities, Brussels, 2001.

Corbetta P., "Metodi di Analisi Multivariata per le Scienze Sociali".Il Mulino, Bologna, 1992.

Cutuli C., "Se scade costa dimeno? Rischi e opportunità della flessibilità in Italia", Franco Angeli, Milano, 2002.

D'addio C. e Rosholm M., Exits from temporary jobs in Europe: A competing risks analysis, Labour Economics, Elsevier, vol. 12(4), pp. 449-468.

Daly, M. and J. Lewis, The concept of social care and the analysis of contemporary welfare states, British Journal of Sociology 51, 2000, pp. 281-298.

Davaki K.(2010). "Benefici del Congedo di Maternità. Parlamento Europeo." Bruxelles.

De Luigi N., Martelli A. (2006). "Il volto sociale della precarietà. Una sfida del welfare locale.". Aracne, Roma.

Del Boca D., Vuri D. (2007), “The Mismatch between Employment and Child Care in Italy: the Impact of Rationing", Journal of Population Economics, 20, 4, pp. 805-32.

D'Odorico L., Salerni L., Cassibba R., Jacob V, Stability and change of material speech to infants from 6 to 20 monthes of age: a longitudinal study of its influence on eary stages of language acquisition, First language, 57, 1997, 313-346.

D’Odorico L., Carubbi S., Salerni N., Calvo V., Vocabulary developement in Italian Children; a lopngitudinal evaluation of quantitative and qualitative aspects, Hournal of Child Language, 28, 2001, pp. 351371. 
D’Odorico L., Lo sviluppo linguistico, Editori Laterza, Roma-Bari, 2010 Eemisch J.F., Purchased Child Care, Optional Family Size and Mother's Employment: Theory and Econometric Analysis, Journal of Population Economics, 2, 2, 1989, pp. 79-102.

Fellini I., La flessibilità a lungo termine dei giovani adulti." In G.A. Micheli (a curadi), Strategie di family formation. Franco Angeli, Milano, 2006

Fullin G., Instabilità del lavoro e vulnerabilità: dimensioni, punti di equilibrio ed elementi di fragilità, Rassegna Italiana di Sociologia, 4, 2002, pp. 553-586

Vivere l'instabilità del lavoro." Il Mulino, Bologna, 2004

Gallando O., Precarietè et Etree dans la vie, Reveu Francaise de Sociologie, 25, 1994, pp. 49-66

Gallino L., Il costo umano della flessibilità, Laterza, Roma-Bari, 2001

Gash, V., Bridge or trap? Temporary worker's transitions to unemployment and to standard employment contract, European Sociological Review, Vol. 5, 2008, pp 651-668

Gash V. E MCGinnity F., Fixed term contracts -the new European inequality? Comparing men and women in west Germany and France, Socio-Economic Review, Vol. 5, 2007, pp. 467-496

Gjerdingen, D. K., P. M. MCGovern, ET AL., Women's postpartum maternity benefits and work experience, Family Medicine, 27, 1995, pp. 592-598.

Gornick, J. C. and M. K. Meyers, Families that Work: Policies for Reconciling Parenthood and Employment, Russell Sage Foundation Publications , New York, 2003

Han, W.-J., C. Ruhm, ET AL, Parental leave policies and parents' employment and leave-taking, Journal of Policy Analysis and Management 28(1), 2009, pp 29-54.

Henderson, A. and L. White, Shrinking welfare states? Comparing maternity leave benefits and child care programs in European Union and North American welfare states, 1985-2000, Journal of European Public Policy 11(3), 2004, pp. 497- 519.

Houston, D. M. and G. Marks, The role of planning and workplace support in returning to work after maternity leave, British Journal of Industrial Relations 41, 2003, pp. 197-214.

Iezzi F. I., Statistica per le Scienze Sociali, dalla progettazione dell'indagine all'analisi dei dati, Carrocci, Roma, 2009

Istat, Quarto rapporto sulla coesione sociale, Centro Istat, Roma, 2013 
Klammer, U., Work life balance from the children's perspective. Children, Changing Families and Welfare States, Edward Elgar, Cheltenham, 2006

Kleinknecht A., Is Labour Market Flexibility Harmful to Innovation?, Cambridge Journal of Economics, 22, 1998, pp. 387-96.

Knight J., Institutions and Social Conflict, Cambridge University Press, Cambridge, 1992

Lamb, M. E., ED., The Role of the Father in Child Development, New Jersey, Wiley, 2004

Longobardi E. (1992) “Funzionecomunicativa del comportamento materno e sviluppo comunicativo-linguistico del bambino nel second anno di vita." Giornale Italiano di Psicologia, 19, 425-448.

Magatti M., Fullin G., Percorsi di lavoro flessibile." Carocci, Roma, 2002.

Micheli G.A., Svantaggi e benefici del lavoro atipico nel confronto tra domanda e offerta, Stato e Mercato, 78, 2006, pp. 437-472.

Migliavacca M., “Famiglie e lavoro. Trasformazioni ed equilibri nell'Europa mediterranea." Bruno Mondadori, Milano, 2008.

Moss, P., From a childcare to a pedagogical discourse - or putting care in its place. Children, Changing Families and Welfare States, J. Lewis. Cheltenham, 2006

Nussbaum, M., Capabilities as fundamental entitlements: Sen and social justice, Feminist Economics (2-3), 2003, pp. 33-59.

Piccone S. (a cura di), Tra un lavoro e l'altro. Vita di coppia nell'Italia postfordista, Carocci, Roma, 2007

Pisati M., La transizione alla vita adulta, Vie Ineguali. Disuguaglianze e corsi di vita nell'Italia contemporanea, Il Mulino, Bologna, 2002

L'analisi dei dati. Tecniche Quantitative per le Scienze Sociali, Il Mulino, Bologna, 2003

Rizza R., Il lavoro mobile, Carocci, Roma, 2003

Salerni N., Calvo V., D’Odorico L., Influenze di ordine affettivo, relazionale e cognitivo nello sviluppo della competenza linguistica, Giornale Italiano di Psicologia, XXVIII(3), 2001, pp. 463-484.

Salmieri L., Coppie flessibili. Progetti di vita quotidiana dei lavoratori atipici, Il Mulino, Bologna, 2006

Saraceno C., Sociologia della Famiglia, Il Mulino, Bologna, 1988

Mutamenti della famiglia e politiche sociali in Italia, Il Mulino, Bologna, 2003. 
Le differenze che contano tra i lavoratori atipici., in S. Bertolini, R. Rizza (a cura di), Atipici?Sociologia del lavoro, 97, 2005, pp. 15-24.

Saraceno C., Naldini, M., Sociologia della famiglia, Il Mulino, Bologna, 2001

Sen, A. Capability and wellbeing. The Quality of Life. M. Nussbaum and A. Sen, Oxford University Press, Oxford, 2001, pp. 9-30

Sennett R., L'uomo flessibile. Le conseguenze del capitalismo sulla vita personale, Feltrinelli, Milano, 1999

Stark R.E., Bornstein, L.E., Demorest M.E., Vocal communication in the first 18 months of life, Journal of Speech and Hearing Research, 36, 1993, pp. 548-558.

Sullivan O., Changing differences by educational attainment in fathers' domestic labour and child care, Sociology 44(4), 2010, pp. 716-732.

Trifiletti R., Dare un genere all'uomo flessibile. Le misurazioni del lavoro femminile nel postfordismo, In Differenze e diseguaglianze. Prospettive per gli studi di genere in Italia. Bologna, Il Mulino, 2003

Tronti L., Ceccato F., Il lavoro atipico in Italia: caratteristiche, diffusione e dinamica, Argomenti, 14, 2005, pp. 27-58.

Vihman M. M., McCune I., When is a word a word?, Journal of child language, 21, 1994, pp. 517-42.

Van Ijzendoorn M.H., Dijkstra J., Bus A.G., Attachemnt intelligence and language: a metanaysis, Social Development, 4, 1995, 115-128.

Waldfogel, J., W.-J. Han, ET Al, The effects of early maternal employment on child cognitive development, Demography 39(2), 2002, pp. 369-392.

Zanetti M.A., "La soggettività del lavoratore flessibile e il problema del consenso alle nuove forme di lavoro." In S. Bertolini, R. Rizza (a cura di), Atipici? Sociologia dellavoro, 97, 122-140. 


\section{Biografia degli autori}

RICCARDO BONATO, nasce nel 1986, laureato in Economia e in Giurisprudenza, si occupa di Diritto del Lavoro e di Sociologia del Diritto. Attualmente è dottorando in Filosofia e Sociologia del Diritto presso l'Università degli Studi di Milano-Bicocca ed è il giuslavorista di riferimento di un giovane studio legale milanese. Referente di numerosi progetti formativi, dirige la collana editoriale Hórisma.

Carlo Botrugno è attualmente dottorando in Diritto e Nuove Tecnologie presso il Centro Interdipartimentale di Ricerca in Storia del diritto, Filosofia e Sociologia del diritto e Informatica giuridica, Università di Bologna. Collabora con la cattedra di Sociologia del diritto presso il Dipartimento di Scienze Giuridiche dell’Università di Bologna.

Simone Camosso è attualmente dottorando in Matematica Pura e Applicata presso il Dipartimento di Matematica dell'Università degli studi di Milano-Bicocca. Analista di formazione, lavora e collabora nello stesso dipartimento nel campo della geometria differenziale e quantizzazione geometrica.

Chiara Caulo, laureata in Psicologia Clinica dello Sviluppo e Neuropsicologia, presso l'Università degli Studi di Milano-Bicocca, attualmente collabora con il gruppo di ricerca e cura dei disturbi del comportamento alimentare in età evolutiva, presso il reparto di Neuropsichiatria Infantile dell' Ospedale San Paolo di Milano.

LEDA RITA CoRRADO, dottoranda di ricerca in Scienze Giuridiche (indirizzo diritto tributario) dell'Università degli Studi di Milano-Bicocca, ho lavorato per le Università di Torino (come cultore della materia), Genova (come docente) e Roma-Tor Vergata (come assegnista di ricerca). I miei studi sono diffusi sulle principali riviste scientifiche del settore. Come giornalista pubblicista, scrivo per varie testate on line di divulgazione giuridica e gestisco un blog su Il Fatto Quotidiano.it. Collaboro con primari studi legali come consulente.

Marianna Nobile è laureata in Filosofia all'Università Vita-Salute San Raffaele. Attualmente è dottoranda di ricerca in Scienze Giuridiche, nell'ambito del curriculum in Filosofia e Sociologia del Diritto, presso il Dipartimento dei Sistemi Giuridici dell'Università degli Studi di Milano-Bicocca, dove è cultore della materia per le cattedre di Filosofia del diritto e di Bioetica. Collabora con il CEF (Comitato per l'Etica di Fine Vita) e fa parte dell'Advisory Board della collana editoriale Hórisma. 


\section{Hórisma}

La collana "Hórisma" si propone di pubblicare e diffondere analisi multidisciplinari - teoriche ed empiriche- di giovani studiosi disponibili a guardare oltre i confini della propria disciplina. L'obiettivo è quello di favorire l'incontro e il confronto su soggetti di interesse collettivo.

La tematica dei volumi sarà selezionata dall'Advisory Board in base alla possibilità di arricchimento multidisciplinare, dando particolare rilevanza agli argomenti di impatto sui giovani. I contributi saranno selezionati da un comitato scientifico costituito in base alle materie interessate.

Direttore di collana:

RicCARDo Bonato -Università degli Studi Milano-Bicocca, Dipartimento di Scienze Giuridiche

Advisory Board:

Giovanni Agostoni -Università degli Studi di Milano, Dipartimento di Beni culturali e ambientali

Simone Bonavita - Università degli Studi di Milano, Dipartimento di Matematica e Informatica

Carlo Botrugno - Università di Bologna, Dipartimento di Scienze Giuridiche

Mario Buatier -Università Bocconi di Milano, Dipartimento di Scienze Economiche e Statistiche

LeDA Rita CoRRADo - Università degli Studi di Milano-Bicocca, Dipartimento di Scienze Giuridiche

Marianna Nobile - Università degli Studi di Milano-Bicocca, Dipartimento di Scienze Giuridiche

Martino Ghielmi - Università Cattolica di Milano, Facoltà di Economia

Maddalena Grigoletto - Università Cattolica di Milano, Facoltà di Economia

Sebastian Saborio - Universidade Federal do Rio de Janeiro, Instituto de Filosofia e Ciências Sociais e Università di Urbino, Dipartimento di Economia, Società, Politica

Olga TARASIUK -University of Hasselt, Centre for Environmental Science 